\title{
High Average Power Harmonic Generation
}

\author{
DAVID EIMERL \\ (Invited Paper)
}

\begin{abstract}
High average power frequency conversion using solid-state nonlinear materials is discussed. Recent laboratory experience and new developments in design concepts show that current technology, a few tens of watts, may be extended by several orders of magnitude. For example, using $K \mathrm{KD}^{*} \mathrm{P}$, efficient doubling ( $>70$ percent) of $\mathrm{Nd}$ : YAG at average powers approaching $100 \mathrm{KW}$ is possible; for doubling to the blue or UV regions the average power may approach $1 \mathrm{MW}$. Configurations using segmented apertures permit essentially unlimited scaling of average power. High average power is achieved by configuring the nonlinear material as a set of thin plates with a large ratio of surface area to volume, and cooling the exposed surfaces with a flowing gas. The design and material fabrication of such a harmonic generator is well within current technology.
\end{abstract}

\section{INTRODUCTION}

$\mathrm{F}$ REQUENCY conversion is a useful technique for extending the utility of high power lasers. It utilizes the nonlinear optical response of an optical medium in intense light fields to generate new frequencies.

Frequency doubling, tripling, and quadrupling generate a single harmonic from a given fundamental high power source. The closely related processes of sum and difference frequency generate also generate a single new wavelength, but require two high power sources. These techniques have been used to generate high power radiation in all spectral regions, from the UV to the far-IR. Optical parametric oscillators and amplifiers generate two waves of lower frequency. They are capable of generating a range of wavelengths from a single frequency source, in some cases spanning the entire visible, and near-infrared regions. The materials used for frequency conversion are often also suitable for other devices, such as electrooptic

Manuscript received July 31, 1986; revised November 21, 1986. This work was supported by the U.S. Department of Energy and the Department of Defense (DARPA), and was performed under the auspices of the U.S. Department of Energy by the Lawrence Livermore National Laboratory under Contract W-7405-ENG-48. This paper was prepared as an account of work sponsored by an agency of the U.S. Government. Neither the U.S. Government nor the University of California nor any of their employees, makes any warranty, expressed or implied, or assumes any legal liability or responsibility for the accuracy, completeness, or usefulness of any information, apparatus, product, or process disclosed, or represents that its use would not infringe privately owned rights. Reference herein to any specific commercial products, process, or service by trade name, trademark, manufacturer, or otherwise, does not necessarily constitute or imply its endorsement, recommendation, or favoring by the U.S. Government or the University of California. The views and opinions of the author expressed herein do not necessarily state or reflect those of the U.S. Government thereof, and shall not be used for advertising or product endorsement purposes.

The author is with the Nonlinear Optical Materials Group, Lawrence Livermore National Laboratory, Livermore, CA 94550.

IEEE Log Number 8613837. switches. A number of excellent reviews of frequency conversion can be found in the literature [30], [44], [26], [52], [31], [3], [47].

These techniques are common for sirgle pulse, or low average power applications, but there has been comparatively little interest in scaling them to high average power. Broadly speaking, this is because the average power in the converted beam is limited primarily by the average power capability of the laser drive. Increasing the average power of a laser generally causes the beam quality to deteriorate, or results in damage to the laser due to the thermal load. Then the primary requirement on the harmonic generator is that it maintains high conversion efficiency for a laser with poor spatial quality. The highest conversion efficiency is then obtained in materials with a large acceptance angle and damage threshold. Although the linear optical absorption in the nonlinear crystal gives rise to thermal gradients which destroy phase matching, managing the heat deposition has been important only if the acceptance angle is very large, that is, under conditions of noncritical phase matching. A second reason for the comparative lack of interest in high average power is that it has been widely assumed that solid-state materials do not handle thermal loads efficiently. Therefore, it has been argued, even if diffraction-limited, high average power lasers were available, frequency conversion using solidstate materials would not be practical.

All of the research to date on average power frequency conversion has addressed frequency doubling second harmonic generation (SHG) of Nd:YAG lasers [24]. Two techniques have been used: intracavity doubling [47], [3], in which the doubler is internal to the optical cavity of the laser, and extracavity doubling, where the doubler is external to the cavity.

In intracavity doubling, the $1064 \mathrm{~nm}$ beam makes many passes through the doubler, and experiences a small conversion efficiency on each pass. One mirror of the cavity is dichroic, and the $532 \mathrm{~nm}$ passes out of the cavity. Geusic [15] reports using (noncritically phase-matched) $\mathrm{Ba}_{2} \mathrm{NaNb}_{5} \mathrm{O}_{15}$ to convert effectively 100 percent of the fundamental to the second harmonic. The average power was nominally $1 \mathrm{~W}$. More recently, Perkins [14], [42] reports using $\mathrm{KTiOPO}_{4}$ for intracavity doubling, obtaining $25 \mathrm{~W}$ of average power.

The first report of average power harmonic generation external to the cavity is by Kogan and Crow [32], who used $\mathrm{KD} * \mathrm{P}$ (potassium dideuterium phosphate) in a type II configuration to double a high quality $10 \mathrm{~Hz}, 1 \mathrm{~J}, 17$ ns Nd: YAG laser. The beam divergence was $0.4 \mathrm{mrad}$ 
and the aperture was $12 \mathrm{~mm}$. They reported an average power of $10.5 \mathrm{~W}$ and a conversion efficiency of about 50 percent. Their system delivered over 20 million shots without any damage to the crystal. (However, the flashlamps were replaced every 5 million shots.) Note that $\mathrm{KD} * \mathrm{P}$ is not noncritically phase matched at this wavelength. More recently, Hon et al. [21], [22], [23] report $35 \mathrm{~W}$ of average power using $\mathrm{CD} * \mathrm{~A}$ (cesium dideuterium phosphate), which does show noncritically phasematching at about $112^{\circ} \mathrm{C}$. Thermal effects are important in $\mathrm{CD}^{*} \mathrm{~A}$ because of its comparatively small temperature bandwidth. The thermal gradients were managed using beam shaping and electrooptic or piezooptic tuning of the crystal. Hon and Moses [40] also report a beam fanning or scanning technique for spreading the thermal load out over a larger volume of crystal, thereby avoiding the large thermal gradients associated with tightly focused beams.

This summarizes the current state of the art in high average power frequency conversion. The average power is relatively modest in part because without a high average power Nd:YAG laser, there is little or no imperative to develop the frequency conversion techniques to handle higher average power. Although the development of high average power solid-state lasers has languished during the last two decades (with $\mathrm{CO}_{2}$ being the prime average power laser) they have recently begun to enjoy a renewed interest. One reason for this is the recent appearance of new applications for lasers, including those associated with military programs, and the Strategic Defense Initiative [13]. The average power requirement of these applications varies up to several orders of magnitude beyond the current state of the art in average power frequency conversion. Also, fusion research at LLNL [34] has shown that commercial power plants based on inertial confinement fusion will require lasers delivering over $1 \mathrm{MJ}$ of short wavelength light at repetition rates of a few hertz. The average power requirement is approximately six orders of magnitude beyond the current state of the art in frequency conversion. This has led to a reexamination of high average power frequency conversion to increase the average power to the required levels.

As part of an overall program addressing the laser for the commercial application of laser fusion, the potential of solid-state lasers to achieve high average power was reexamined recently at LLNL [13]. New design concepts and recent developments in materials and techniques provided a focus for that study. Particular attention was paid to thermal management and optical distortion, and especially to efficient utilization of the pump energy. The conclusion was that solid-state lasers are capable of unconstrained scaling of average power, while maintaining high beam quality. Moreover, it was found that efficiencies of over 10 percent should be achieved at high power and irradiance. The study showed how this is possible with careful attention to conserving the pump energy and intelligent thermal management. In parallel with this study, and using experience gained in frequency conversion of a $100 \mathrm{KJ}, 100 \mathrm{TW}$ laser [48], [46], the potential of solid- state nonlinear materials to reach high average power while maintaining high conversion efficiency was also thoroughly evaluated. The conclusion was that solid-state nonlinear materials also support unlimited average power, at conversion efficiencies in excess of 70 percent. This is possible with careful attention to the thermal gradients and the beam shape, and balancing the various sources of phase mismatch. For example, using $\mathrm{KD} * \mathrm{P}$ in a quadrature configuration to double a $10 \mathrm{~ns} \mathrm{Nd}$ : YAG laser permits over $50 \mathrm{KW}$ of average power in a single crystal segment to be doubled with an efficiency in excess of 70 percent.

This paper reports the major results of the study of high average power frequency doubling. Single pulse frequency conversion is summarized in Section II, which includes some perhaps unfamiliar results on the role of dephasing at high conversion efficiency. Thermal gradients are analyzed in Section III. The performance of the scalable design (the $N$-plate design) is fully analyzed in Sections IV and V, and design procedures are given in Section VI.

Although this paper treats frequency doubling, by a trivial extension it also addresses third harmonic generation, sum and difference frequency generation, and optical parametric oscillator (OPO) design. It appears that all these other processes are scalable in average power just as second-harmonic generation (SHG) is. Consequently, high average power solid-state laser systems are just as capable of frequency versatility as are single pulse systems.

\section{Single Pulse Conversion Efficiency}

The equations describing the interaction of the first and second harmonics are

$$
\begin{aligned}
& \partial E_{2} / \partial z=C E_{1}^{2} e^{i \Delta k z} \\
& \partial E_{1} / \partial z=-C E_{2} E_{1}^{*} e^{-i \Delta k z} .
\end{aligned}
$$

Here, the fields are normalized so that the intensity is $E^{2}$ with no other factors and the coupling $C$ is

$$
C=5.46 d_{\text {eff }}(\mathrm{pm} / \mathrm{V}) / \lambda_{1}(\mu \mathrm{m})\left(n_{1} n_{2} n_{3}\right)^{1 / 2}
$$

where the units of $d_{\text {eff }}$ and $\lambda$ are as indicated, and the units of $C$ are $G W^{-1 / 2}$. These equations have been solved by Armstrong, et al. [2]. For a crystal of thickness $L$, the conversion efficiency is

$$
\eta=\tanh ^{2}\left[\frac{1}{2} \tanh ^{-1}\left(\operatorname{sn}\left[2 \eta_{o}^{1 / 2}, 1+\delta^{2} / 4 \eta_{o}\right]\right)\right]
$$

where

$$
\begin{aligned}
\eta_{0} & =C^{2} I L^{2} \\
\delta & =(1 / 2) \Delta k L
\end{aligned}
$$

and $s n$ is a Jacobi elliptic function. These parameters are the nonlinear drive and half the difference in phase between the second harmonic waves originating at the input and exit faces of the crystal. This dephasing (which is 


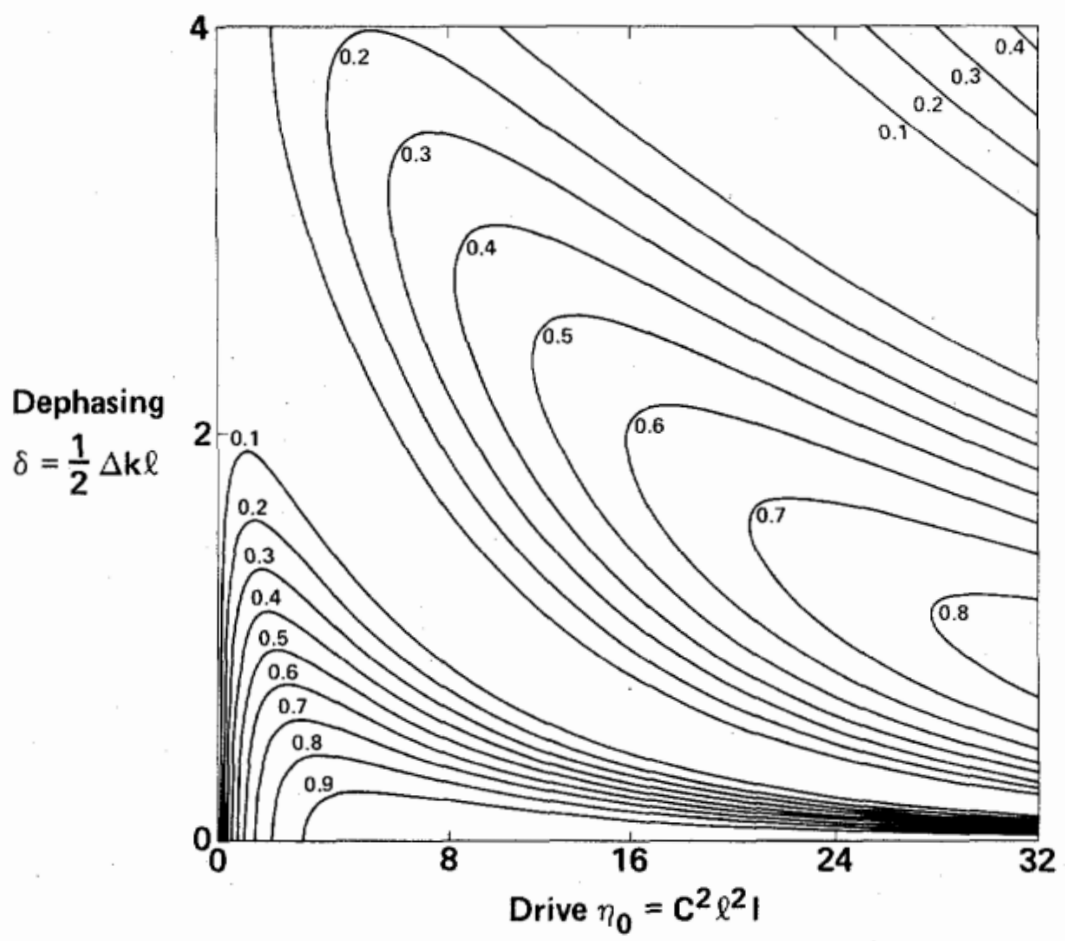

Fig. 1. Contour plot of the conversion efficiency $\eta$ as a function of the (dimensionless) drive $C^{2} l^{2} I$ and the dephasing $(1 / 2) \Delta k l$. The line of zeros between the first and second lobes follows the approximate curve $\delta=8 \eta_{0}^{1 / 2} \exp \left(-\eta_{0}^{1 / 2}\right)$. Conversion using the first lobe becomes increasingly sensitive to detuning at higher drive.

proportional to the phase mismatch) depends primarily on the local beam direction and the temperature variation in the crystal, but it also receives contributions from any effect which disturbs the indexes of refraction. Thus, besides the crystal orientation and the laser beam quality, it also includes the effects of scattering, diffraction, optical aberrations and defects, and thermal gradients and stresses. The conversion efficiency depends on the total dephasing, but in many applications only the beam divergence $\delta \theta$ is significant.

$$
\Delta k=\beta_{\theta} \delta \theta+\beta^{\prime \prime} \delta \theta^{2} .
$$

For critically phase-matched materials the first term dominates and $\Delta k$ is linear in $\delta \theta$. For noncritically phasematched materials, the first term vanishes and $\Delta k$ is quadratic in $\delta \theta$. However, note that the dependence of $\eta$ on $\eta_{o}$ and $\delta$ is the same for both critically and noncritically phase-matched materials. The design procedure for optimizing the crystal length by trading dephasing against drive is the same for both the critically and noncritically phase-matched cases. If $\eta_{o}<1$, then the conversion efficiency is

$$
\eta=\eta_{o}(\sin \delta / \delta)^{2} \text {. }
$$

This shows that if the drive is small, then it is also a direct measure of the phase-matched conversion efficiency. For larger drives the fundamental pulse is more strongly depleted, and the point $\eta_{o}=1 \mathrm{can}$ be considered as the point where saturation begins. For example, the conversion efficiency for $\delta=0$ is

$$
\eta=\tanh ^{2}\left(\eta_{0}^{1 / 2}\right)
$$

and $\eta$ approaches 1 as the drive tends to infinity.

A second and less well-known effect is that the sensitivity of the conversion process to the dephasing $\delta$ increases as the drive increases [9], [10], [25]. The effective dephasing $\delta_{\text {eff }}$ is defined as follows:

$$
\delta_{\text {eff }}=\delta \exp \left(\sqrt{\eta_{0}}\right)>\delta .
$$

The saturating efficiency $\eta$, for $\eta_{0} \gg 1$, and $\delta_{\text {eff }}<\pi$, is approximately

$$
\eta=\left(\tanh \left(\sqrt{\eta_{0}}\right) \sin \delta_{\text {eff }} / \delta_{\text {eff }}\right)^{2} .
$$

The exact saturating behavior, as a function of $\eta_{0}$ and $\delta$, is presented as a contour map in Fig. 1 . The lines connect points $\left(\eta_{0}, \delta\right)$ which give the same saturating efficiency; there are nine contours corresponding to the efficiencies $0.1,0.2,0.3, \cdots, 0.8,0.9(\eta=1$ does not exist). It is clear that any energy in the laser pulse which meets the crystal with an $\eta_{0}$ and $\delta$ for which the conversion efficiency is low is not converted. Therefore, high conversion efficiency requires that all the energy in the laser pulse meet the crystal at or above a high efficiency contour. Efficient conversion requires using either the central lobe of Fig. 1 (the lobe closest to the origin), or the secondary lobe. For the central lobe, efficient conversion requires that

$$
|\delta| \ll \pi / 10
$$

and

$$
1<\eta_{o}<5
$$




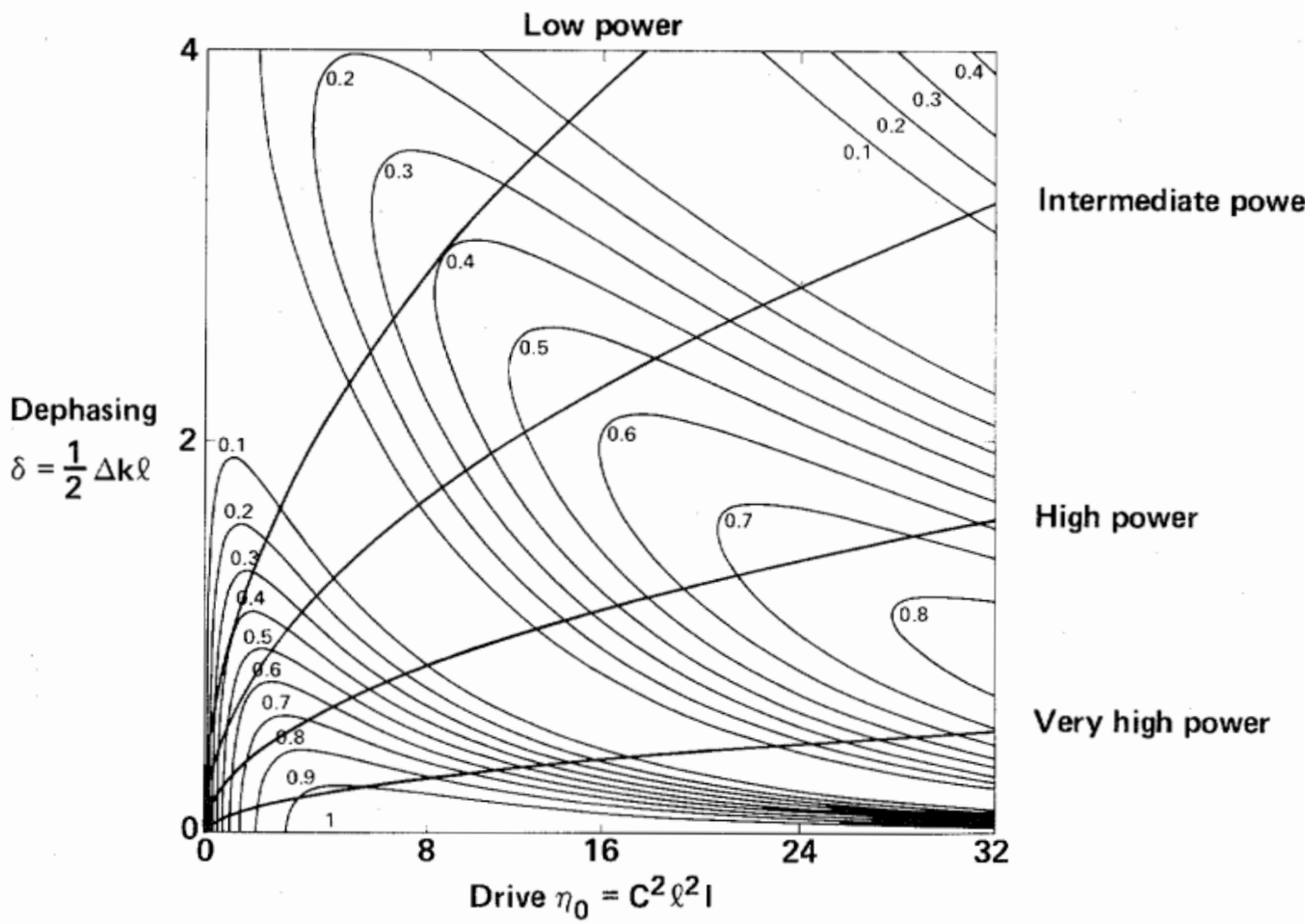

Fig. 2. The crystal thickness is chosen by optimizing the tradeoff between drive and dephasing. For fixed intensity and beam divergence, the system variables lie on parabolas in the drive/dephasing plane. Each point on a parabola corresponds to a particular crystal thickness. The crystal thickness is chosen to give peak efficiency, which requires operating in the central lobe close to the origin, as discussed in the text. By optimizing the crystal length this way, the conversion efficiency is determined entirely by the total peak power in the laser pulse and the beam quality. The efficiency is not optimized by maximizing the intensity, but by optimizing the peak power per transverse mode. See text for details.

The secondary lobe requires that

$$
\delta=1.5 \pm \pi / 10
$$

and

$$
15<\eta_{o}<40
$$

Clearly, the maximum efficiency in the secondary lobe is less than that in the central lobe. The efficiency achieved depends on the details of the spatial and temporal character of the drive. This is especially important at the edges of the pulse where the intensity drops to zero. The central lobe converts this light moderately efficiently, but the secondary lobe is bounded by a line of zeros (cf. the point $\eta_{o}=12, \delta=1.5$ ). Consequently, using the secondary lobe necessarily implies poor efficiency for the spatial and temporal edges of the beam.

For Gaussian pulses, this causes a significant reduction in conversion efficiency. Only for pulses which are flat in space and time will the conversion efficiency be high in the secondary lobe. While for some pulse shapes this secondary lobe may be more efficient than the central lobe, for most applications the central lobe will give higher efficiency. Therefore, without dismissing the secondary lobe, we focus on the central lobe in this study.

The choice of crystal thickness is illustrated in Fig. 2. If the intensity and the angular divergence of the beam are considered to be fixed, then as the crystal thickness is varied, a curve is mapped out in the drive/dephasing plane of Fig. 1. The drive varies quadratically with the crystal thickness, whereas the dephasing varies linearly; the curve is therefore a parabola. For high intensity beams, the parabola lies close to the drive axis, and as the intensity is lowered it becomes more vertical, as shown. The conversion efficiency varies under the curve as indicated by the contours. As the crystal length is increased, the efficiency peaks and then oscillates between the peak and zero, in a nonsinusoidal fashion. The optimum crystal length is the lowest length for which the efficiency peaks. As discussed above, this is always in the central lobe of the efficiency contour. Thus, for a given intensity and angular divergence, there is an optimum crystal length, which depends on the tradeoff between drive and dephasing.

It is still true that even for crystals whose length is optimal, high efficiency requires that the intensity be larger than some value defined by the angular divergence of the beam. It has often been argued in the literature that the efficiency will be maximized by going to as high an intensity as possible without damaging the crystal. This leads to system designs which focus the beam into the nonlinear crystal. However, proper optimization of the crystal and system parameters shows that this argument is incorrect. and that the performance is not in fact opti- 
mized by maximizing the intensity. We shall now prove this somewhat counterintuitive result.

Consider a laser beam with a fixed peak power incident on a critically phasematched crystal, and allow the area of the beam to be adjustable using telescopes. As the (de)magnification $m$ is changed, the intensity and angular divergence change, and therefore the optimal crystal thickness changes, also. If the drive is telescoped down by $m$ to increase the intensity, then the intensity increases by $m^{2}$ and the optimum crystal length decreases by $m$. The drive to the crystal remains unchanged, and is independent of the demagnification. Moreover, the angular divergence increases by $m$ also, so that the product $L \delta \theta$ is independent of $m$. Telescoping the beam has no effect on either the drive or the dephasing, and the conversion efficiency is independent of the demagnification. Telescoping merely changes the crystal size; it has no effect on the performance. On the other hand, the fluence on the crystal increases as $m^{2}$. There is therefore a minimum beam area or crystal size fixed by the optical damage threshold of the crystal. So long as the crystal size and beam area are large enough to keep the fluence below the damage limit, all beam areas are equivalent in performance; there is no advantage whatsoever in maximizing the intensity.

Mathematically, the drive on the crystal is

$$
\eta_{o}=C^{2} L^{2} I=C^{2}\left(L^{2} / H H^{\prime}\right) P_{\mathrm{pk}}
$$

where the beam area is $H H^{\prime}$ and $P_{\mathrm{pk}}$ is the peak power in the laser pulse (Fig. 3). Clearly doubling all the dimensions of the crystal and beam area leaves the drive unchanged. The dephasing may be expressed in terms of the beam quality, $Q$, as follows:

$$
\delta=\frac{1}{2} \Delta k L=\frac{1}{2}\left(\beta_{\theta} \lambda\right) \cdot Q(L / H)
$$

where the beam quality $Q$ is given by

$$
\delta \theta=Q(\lambda / H) \text {. }
$$

$Q$ is roughly the ratio of the beam divergence in the sensitive direction of the crystal to the beam divergence of a diffraction-limited beam of the same area, and it is independent of the beam dimensions (see Fig. 3). Clearly doubling all the dimensions leaves the dephasing unchanged. Therefore the drive, dephasing, and efficiency are independent of the beam size. By eliminating the crystal thickness from (16) and (17), the condition for high efficiency is obtained:

$$
P_{\mathrm{pk}}=E / \tau \geq\left(\beta_{\theta} \lambda / C\right)^{2}\left(\eta_{o} / 4 \delta^{2}\right) \cdot Q^{2}
$$

where $E$ is the pulse energy. The ratio $\eta_{o} / \delta^{2}$ is independent of the crystal length and is chosen to give high efficiency. Then this condition, (19), gives the peak power required to reach high conversion efficiency, regardless of beam size or crystal length. It increases rapidly as the beam quality deteriorates. This condition can also be viewed as a yes/no test for using a particular material to double the frequency of a given laser system. Note that a poor material will be poor regardless of the demagnification, and cannot be "tweaked up" to give high efficiency.

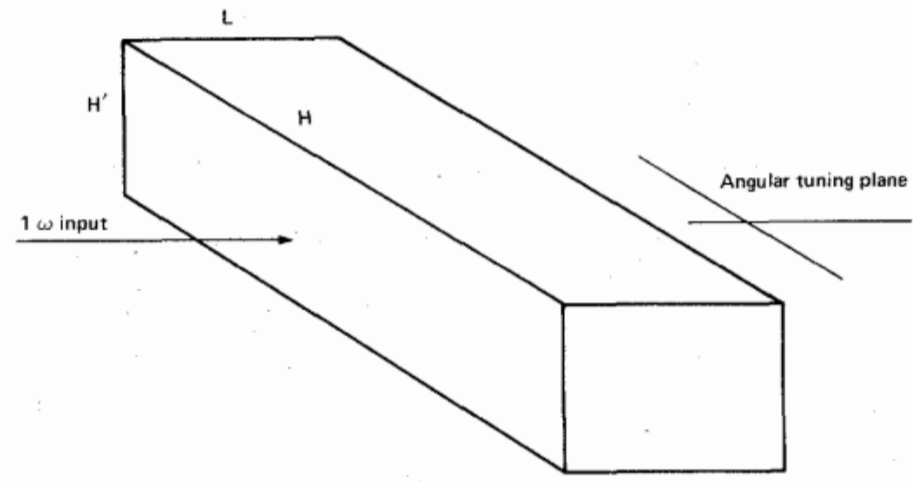

Fig. 3. The crystal dimensions of the harmonic generator. The beam area is $H H^{\prime}$, and the sensitive direction of the crystal for angular misalignments is the $(L H)$ plane. The performance of the generator is independent of the size of the crystal and depends only on its shape.

The same analysis can be carried through for noncritically phase-matched materials. In this case the dephasing $\delta$ is quadratic in the beam divergence $\delta \theta$. The ratio $\eta_{o} / \delta^{2}$ is independent of the crystal length but varies inversely as the fourth power of the beam quality, $Q$, rather than the second power as in the case of critically phase-matched materials. Having chosen the operating point in the drive/ dephasing plane (Fig. 2) this ratio $\left(\eta_{o} / \delta^{2}\right)$ is then fixed, and on being reexpressed in terms of the laser parameters, it gives the peak laser power required to operate at that point with the given laser divergence and nonlinear material. This condition on the peak power, analogous to (19) for critically phase-matched materials, is

$$
P_{\mathrm{pk}}=\left(\beta^{\prime \prime} \lambda / C\right)^{2}\left(\eta_{o} / \delta^{2}\right) Q^{2}\left(\delta \theta^{2}\right) .
$$

Evidently, the peak power required for efficient conversion goes down as the beam becomes more collimated. Thus, as the beam aperture is increased by telescoping, the peak power required decreases. In principle, it is possible to reduce the peak power requirement to arbitrarily small values, by using crystals as large as necessary to give the required beam collimation. Again, we find the counterintuitive conclusion that lower peak power is needed for larger beam apertures; focusing into noncritically phase-matched materials gives a higher peak power requirement than telescoping the beam up to larger aperture. This argument can also be restated as a crystal size requirement. High conversion efficiency is possible, in principle, in noncritically phase-matched materials regardless of the peak power of the laser, simply by arranging for a sufficiently large beam aperture and therefore low beam divergence. In practice, the availability of high quality optics and crystals in larger sizes determines the applicability of this scaling argument.

Another corollary of this analysis is that one can in principle simply design around the optical damage problem, by choosing a beam area which is sufficiently large. A material which meets the efficiency condition will be efficient at beam sizes where the fluence is arbitrarily low. Surmounting optical damage problems only requires crystals of sufficient size. This conclusion has profound 
consequences for design and performance of nonlinear optical devices.

\section{General Average Power Considerations: The $N$-P LATE Design}

If the linear optical absorption coefficient is $\alpha_{l}$, then the heat deposited per unit volume in the crystal is

$$
W=\alpha_{I} I_{\mathrm{av}}=\alpha_{I} f \tau I_{\mathrm{pk}} .
$$

The heat deposition is independent of position if, and only if, the optical absorption is the same at the drive and converted frequencies. This is certainly not true for most materials, but for the moment we shall continue with (21) as a useful approximation and leave the question of the frequency dependence of the absorption until later. With a uniform heat deposition, a steady state is set up in which temperature gradients are established to carry off the heat by thermal conduction.

Whatever the direction of the temperature gradients relative to the direction of the laser pulses, the temperature profile set up in the crystal will be quadratic. We have

$$
\nabla^{2} T=W / K
$$

where $K$ is the thermal conductivity (assumed isotropic). Recall that a streamline for the heat flow is a line which is always parallel to the local direction of heat flow, or grad $T$. Along any streamline the temperature is quadratic:

$$
T=T_{0}+s T_{1}+s^{2} W / 2 K .
$$

Here, $s$ is the distance along the streamline and $T_{0}$ and $T_{1}$ are integration constants.

Consider the point in the crystal where the temperature in steady state is a maximum and consider the streamlines emanating from that point. For all such streamlines $T_{1}$ is zero because the temperature gradient is zero at the maximum:

$$
T(s)=T_{0}+s^{2} W / 2 K .
$$

Every streamline originates at the warmest point and eventually meets the boundary of the crystal where the heat it transports is carried off by some agent other than thermal conduction. If a streamline has length $s$ then the temperature difference between its ends is simply

$$
\Delta T=s^{2} W / 2 K \text {. }
$$

The lowest temperature in the crystal is therefore at the other end of the longest streamline. The temperature difference between the warmest and coolest point in the crystal is therefore minimized by arranging for the longest streamline to be as short as possible. This rather obvious conclusion has profound consequences for high average power lasers.

Because optical materials damage at high fluence, the only way to achieve high energy per pulse is to increase the aperture of the laser. High pulse energy and high average power will be achieved with large apertures. If the

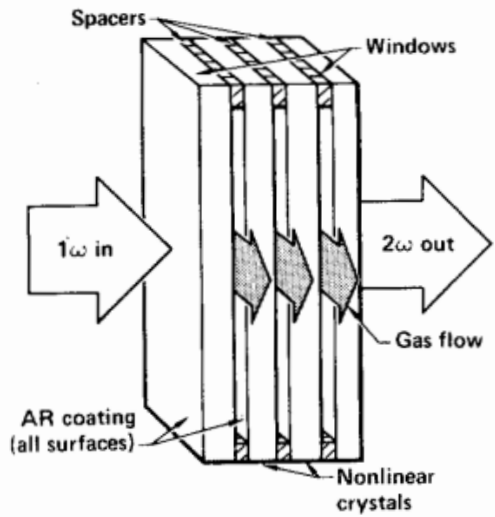

Fig. 4. A high average power harmonic generator design with efficient scalable thermal management.

streamlines are perpendicular to the laser beam, then increasing the average power by increasing the aperture causes the length of the streamlines (and therefore the maximum temperature difference) to increase. On the other hand, if the streamlines are parallel to the laser beam, their length is fixed by the crystal thickness, and the temperature difference is independent of the aperture. The total average power is then not limited by thermal considerations, although the average power per unit area is.

With the streamlines parallel to the laser beam, the temperature is a maximum in the center of the crystal and a minimum on its faces. The temperature difference between the center and the faces is

$$
\Delta T=L^{2} \alpha_{I} I_{\mathrm{av}} / 8 K .
$$

Typically, $\Delta T$ is limited by the thermal limits discussed in the next section. Then the average power per unit area is maximized by choosing thin crystals. However, thin crystals do not convert efficiently. On comparing $\Delta T$ with the drive $\eta_{0}$, we find that $\eta_{0}$ and $\Delta T$ are both proportional to $L^{2}$, and that their ratio is determined solely by the product $f \tau$, independent of the crystal thickness:

$$
\Delta T / \eta_{0}=f \tau\left(\alpha_{I} / 8 K C^{2}\right) .
$$

The only system variables in this equation occur in the product $f \tau$; the other variables are either material constants or design parameters fixed by performance and/or damage limits.

For a single crystal plate, (27) shows that the duty cycle $f \tau$ is fixed by the thermal limits on $\Delta T$ and high conversion efficiency and not the crystal thickness. The reason is that $\Delta T$ determines the product $I_{\mathrm{av}} L^{2}$, whereas $\eta_{0}$ determines the product $I L^{2}$. These two conditions can be met only if $I$ and $I_{\mathrm{av}}$ have the correct ratio; hence the condition on the duty cycle, $f \tau$.

The thermal streamlines can be shortened further by replacing the single crystal with a set of $N$ thinner crystals with the same total thickness and flowing a coolant gas between them (see Fig. 4). This is equivalent to removing heat from the single crystal system on planes inside the crystal. The temperature difference within any single plate 
is reduced by a factor $N^{2}$, and therefore the average power per unit area is increased by the same factor for the same limit on $\Delta T$. If the thickness of a single plate is $l=L / N$, then

$$
\Delta T=l^{2} \alpha_{I} I_{\mathrm{av}} / 8 K
$$

and

$$
\Delta T / \eta_{0}=f \tau / N^{2}\left(\alpha_{I} / 8 K C^{2}\right)
$$

Thus for a given limit on $\Delta T$, the $N$-plate design allows the duty cycle $f \tau$ to be a factor of $N^{2}$ larger and delivers an average power per unit area which is larger by the same factor.

\section{The Sources of Dephasing}

Temperature gradients cause thermal stresses to be established in the medium. They also give rise to inhomogeneities in the refractive indexes; these arise directly, through $d n / d T$, and also indirectly, through the stress dependence of the indexes. There are also contributions from turbulence and optical dispersion in the coolant gas. We consider each one of these effects in turn.

In general the phase mismatch $\chi(z)$ for a single plate is the sum of three terms:

$$
\begin{aligned}
\chi(z) & =\int_{0}^{z} \Delta k d z \\
& =\phi_{g}+\beta_{\theta} \delta \theta z+\beta_{t} \int_{0}^{z}\left(T\left(z^{\prime}\right)-T_{0}\right) d z^{\prime}
\end{aligned}
$$

where $\phi_{g}$ is the phase mismatch at the input face. The crystal is taken to be oriented so that for a perfect plane wave input $(\delta \theta=0)$, the harmonic generation process is exactly phasematched at the mean operating temperature of the plate, $T_{0}$. Then the last term in (30) includes only the effect of deviations from the mean (or operating) temperature of the plate.

\section{Optical Dispersion}

The phase $\phi_{g}$ is the phase difference between the waves at the entrance surfaces of the first plate and the $M$ th plate. Thus, besides the phases associated with propagation and nonlinear optics, they include the phase accumulated in the gas coolant flowing between the plates. This is significant because the optical dispersion in the coolant disturbs the relative phases and can reduce the conversion efficiency. If the separation between the plates is $l_{s}$ then the phase accumulated at the entrance to the $M$ th plate is

$$
\chi(M)=\left(\omega_{3} n_{3}-\omega_{2} n_{2}-\omega_{1} n_{1}\right) l_{s}(M-1) / c
$$

where the indexes now refer to the coolant and not the crystal. If the phase $\chi(M)$ is $\pi$, then the harmonic generated in the $M$ th plate has a phase of $\pi$ relative to the harmonic generated in the first plate. The harmonics from all the plates will not add constructively unless $\chi(N)$ $\ll \pi$. Constructive interference requires that

$$
l_{s}<l_{c} /(N-1)
$$

TABLE I

COHERENCE LENGTHS OF GASES ${ }^{\mathrm{a}, \mathrm{b}}$

\begin{tabular}{lc}
\hline \hline Gas & $l_{c}(\mathrm{~cm})$ \\
\hline $\mathrm{He}$ & 42.76 \\
$\mathrm{Ne}$ & 22.90 \\
$\mathrm{Ar}$ & 2.305 \\
$\mathrm{Kr}$ & 1.031 \\
$\mathrm{Xe}$ & 0.4407
\end{tabular}

\begin{tabular}{ll}
$\mathrm{H}_{2}$ & 3.289 \\
$\mathrm{~N}_{2}$ & 2.153 \\
$\mathrm{O}_{2}$ & 1.787 \\
$\mathrm{CO}$ & 1.239 \\
$\mathrm{CO}_{2}$ & 1.019 \\
$\mathrm{CH}_{4}$ & 0.9773 \\
$\mathrm{SF}_{6}$ & 0.8763 \\
$\mathrm{Air}$ & 2.083 \\
\hline \multicolumn{2}{c}{${ }^{\mathrm{a}} 1.064 \mu$ m and STP. } \\
'belsko and Eimerl [50].
\end{tabular}

where $l_{c}$ is the coherence length of the coolant

$$
l_{c}=c /\left(\omega_{3} n_{3}-\omega_{2} n_{2}-\omega_{1} n_{1}\right) .
$$

The index difference between the waves in nitrogen at STP is $3.9 \times 10^{-6}$, so that the coherence length for doubling $1 \mu \mathrm{m}$ is about $2.1 \mathrm{~cm}$. A recent study by Velsko and Eimerl [50] measured these lengths directly using a nonlinear interferometer, to an accuracy better than that of 0.5 percent. These data are listed in Table I. It is clear that coolant dispersion is a significant aspect of high average power design.

\section{Angular Dephasing}

The second term in the phase-mismatch is the angle tuning term. This term originates in the angular misalignment of the plate(s) and in the spatial harmonics in the drive. It accumulates with each plate. For example, at low drive, the condition $\Delta k \cdot L=\pi$ causes the harmonic from the last plate to be almost exactly out of phase with that from the first plate. At high power, this destructive interference occurs even for $\Delta k \cdot L \ll \pi$ (see Fig. 1). With regard to angular misalignment, the $N$-plates behave like a single plate of the same total thickness. We can then estimate from Fig. 1 that the right-hand side of this inequality must be about $\pi / 10$. Therefore, angular detuning limits the total plate thickness $L=N l$ :

$$
\Delta k \cdot L=\beta_{\theta} \delta \theta N l \ll \pi / 10 .
$$

Here, $\delta \theta$ contains the error in orientation of the crystal plates, as well as the beam structure effects. Other things being equal, it might be expected to increase in an rms fashion with the number of plates.

\section{Longitudinal Temperature Rise}

The third term in the phase mismatch is the temperature dephasing term. In this expression $T_{0}$ is the temperature at which the process is exactly phase matched, and the 
plate is oriented so that $T_{0}$ is also the mean temperature of the plate. Then the complete temperature dephasing integral is zero for each plate

$$
0=\int_{0}^{l}\left(T\left(z^{\prime}\right)-T_{0}\right) \delta z^{\prime}
$$

In contrast to the other two terms, the complete temperature dephasing term is exactly zero for each plate; it does not accumulate with the number of plates. To ensure high conversion efficiency, it is sufficient to limit the maximum phase mismatch in a single plate.

The effect of the parabolic temperature profile on the conversion efficiency cannot be expressed in closed mathematical form. However, in the approximation that the temperature profile is two step functions, the conversion efficiency can be obtained using the effective phase mismatch, $\chi_{t}$ :

$$
\chi_{t}=\frac{1}{8} \beta_{t} \Delta T l .
$$

High conversion efficiency requires that $\chi_{t}<\pi / 10$, as before. The appropriate temperature detuning limit is

$$
\beta_{t} \Delta T l<4 \pi / 5 \text {. }
$$

The step function approximation certainly gives an upper limit on the effective dephasing, and (37) is a conservative limit on $l \Delta T$.

\section{Transverse Temperature Rise}

The temperature of the crystal faces is not strictly uniform over the entire face (Fig. 5). The face is cooled by flowing gas, which warms as it traverses the crystals and removes heat from them. In steady-state flow the coolant removes a heat flux $\mathrm{Wl} / 2$ from each of the two faces on either side of it. The coolant temperature rises approximately linearly as it absorbs heat from the crystals, and its gradient is [29]

$$
d T / d x=W l / C_{p} v b
$$

where $C_{p}$ is the specific heat per unit volume of the coolant, $v$ is its velocity, and $b$ is the thickness of the flow layer absorbing the heat.

The temperature of the faces and the coolant are determined by the heat flow

$$
W l / 2=h\left(T_{c}-T_{f}\right)
$$

where $h$ is the convective heat transfer coefficient and $T_{c}$ and $T_{f}$ are the temperatures of the crystal faces and the mixed mean temperature of the coolant. Now the heat removed per unit area $(W l)$ is independent of $x$, and if $h$ is independent of $x$ the temperature gradients of both the coolant and the crystal in the $z$-direction are also independent of $x$. This implies that if the temperature of the coolant rises by $\Delta T_{\mathrm{tr}}$ as it traverses the crystal face, then the surface temperature of the crystal also rises by the same amount, to maintain the same rate of heat removal from the crystal. Now the transverse temperature difference

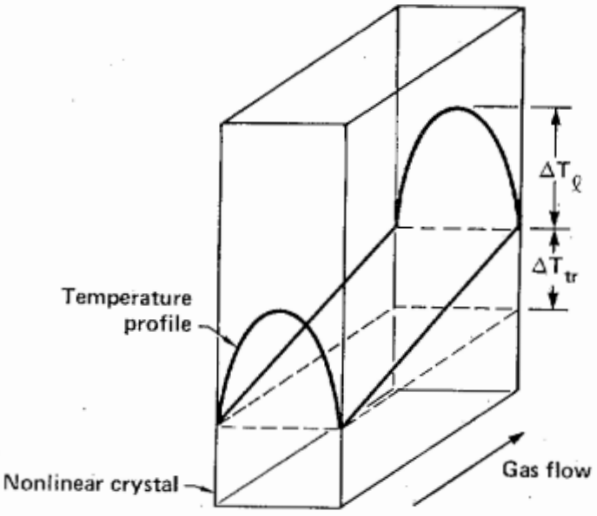

Fig. 5. The temperature profile in a single plate. The longitudinal temperature profile (the parabola) conducts the absorbed heat out to the surfaces. The coolant temperature rises as it carries away the heat, causing a transverse temperature gradient. Both temperature differences are important in optimizing the performance.

$\Delta T_{\text {tr }}$ must be small enough to lie within the temperature bandwidth of the phase-matching condition. If this condition is not met by $\Delta T_{\mathrm{tr}}$, then it will not be possible to achieve phase matching over the entire aperture.

The difference between the absolute maximum and minimum of the temperature in the crystal is $\Delta T+\Delta T_{\mathrm{tr}}$. The full temperature dephasing limit is therefore

$$
\beta_{t} l\left(\Delta T_{\mathrm{tr}}+1 / 8 \Delta T\right)<\pi / 10
$$

where the factor of $1 / 8$ derives from the averaging of the longitudinal temperature dephasing, as in (36).

The transverse temperature rise $\Delta T_{\mathrm{tr}}$ is obtained by integrating (38) over the aperture, $H$, and is simply related to the longitudinal temperature difference, $\Delta T$, given by (28). Their ratio is essentially the ratio of the heat flow resistances of the crystal and the gas coolant

$$
\Delta T_{\mathrm{tr}} / \Delta T=\left(8 K / C_{p} v b\right) \cdot(H / l) .
$$

The transverse temperature rise will be larger unless

$$
C_{p} v b / K>8 H / l \text {. }
$$

This is not a trivial condition to meet, as the following estimate shows. The ratio $H / l$ is fixed by mechanical considerations to be not more than about 15 , and the specific heat $C_{p}$ can be estimated using the perfect gas result at STP, $C_{p}=555 \mathrm{~J} / \mathrm{m}^{3} / \mathrm{K}$. For the crystal conductivity, we use the value for $\mathrm{KDP}, 2 \mathrm{~W} / \mathrm{m} / \mathrm{K}$. Then the inequality states that

$$
v b>0.43 \mathrm{~m}^{2} / \mathrm{s} .
$$

The length $b$ must be less than or equal to the separation between the crystals and if this is taken to be $2.2 \mathrm{~mm}$ the flow velocity is $200 \mathrm{~m} / \mathrm{s}$, which is comparable to the velocity of sound. The flow must be turbulent to achieve this value for $b$. If the flow is laminar, then $b$ is the boundary layer thickness (determined by the thermal conductivity of the gas) and is much smaller. The flow velocity can be reduced somewhat by increasing the pressure, and reducing the temperature of the gas. However, it appears cer- 
tain that reducing the transverse temperature rise below the longitudinal rise requires high mass flow cooling.

Secondary aspects of such high mass transfer flows include the possibility of exciting acoustic modes in the crystals and the possibility of damaging the crystal surfaces by impact of solid microparticles in the coolant gas. These will cause the fracture strength to decrease and may reduce the optical quality of the output.

Finally we note that the transverse temperature gradient generates a transverse flow of heat. The ratio of the transverse and longitudinal heat currents is

$$
q_{x} / q_{z}=\left(K_{x x} / K_{z z}\right)\left(\Delta T_{\mathrm{tr}} / \Delta T\right)(l / H) .
$$

Clearly the transverse temperature gradient is smaller. Therefore, under appropriate flow cooling conditions, the temperature gradient is indeed essentially parallel to the laser beam.

\section{Cooling Rate}

The temperature difference between the crystal and the coolant is determined by the convective heat transfer coefficient $h$ :

$$
T_{c}-T_{f}=W l / 2 h=\Delta T_{b l} .
$$

This temperature difference is limited only by the thermal or chemical stability regions of the crystal. As examples, consider the most common materials which are available in large sizes. $\mathrm{KD} * \mathrm{P}$ and lithium iodate begin to decompose below $200^{\circ} \mathrm{C}$, whereas lithium niobate and $\mathrm{CD} * \mathrm{~A}$ are stable up to the maximum temperature at which they can be phase matched, around $120^{\circ} \mathrm{C}$. Consequently, the crystal temperature and the cooling rate are limited. The average power density is also limited:

$$
I_{\text {av }} \leq h\left(T_{c}-T_{f}\right) / \alpha_{l} l
$$

Typical values for the parameters in this equation are $h$ $\sim 1 \mathrm{KW} / \mathrm{m}^{2} \mathrm{~K}, 10 \mathrm{KW} / \mathrm{cm}^{2}$. Under the exected operating conditions, flow cooling is easily capable of removing the heat deposited in the crystals.

\section{Beam Quality and Thermal Loading}

The temperature profile of the crystal is determined both by the heat loading $W$ and the flow cooling condition. The heat loading is due entirely to optical absorption, and therefore has the same spatial structure as the average power density. If this is spatially nonuniform, the temperature of the crystal will tend to have the same spatial variation as average power density.

In this way spatial variations in the beam profile translate directly into a transverse temperature variation. This argument applies to any spatial variation in intensity which is systematically repeated from pulse to pulse. It therefore applies to the energy at the edge of the beam where the intensity is dropping to zero, as well as to small-scale variations.

The transverse heat flow can be calculated by separation of variables in the diffusion equation, letting the lon- gitudinal temperature profile be determined by the deviations of the heat loading from its average value. Then, in the transverse direction

$$
(\Delta T)_{x x}=W / K(\Delta I(x) / I)
$$

where $W$ and $I$ are spatial averages. Decomposing $\Delta T$ and $\Delta I$ into Fourier components, we find

$$
\Delta T(\Lambda)=\left(W \Lambda^{2} / 4 K \pi^{2}\right)(\Delta I(\Lambda) / I)
$$

where $\Lambda$ is the spatial wavelength of the heat loading. In terms of the longitudinal temperature difference $\Delta T$

$$
\Delta T(\Lambda) / \Delta T=\left(2 \Lambda^{2} / \pi^{2} l^{2}\right)(\Delta I(\Lambda) / I) .
$$

As long as the periodicity $\Lambda$ is comparable to or smaller than the plate thickness, the transverse variation in temperature will be small compared to the longitudinal temperature variation.

The problem of the temperature profile at the edges of the beam can be solved in at least two ways. One way is to arrange for the fill factor of the crystal aperture to be as close to unity as possible and to thermally insulate the edges. The temperature drop at the edge will be smaller than the longitudinal $\Delta T$ if the beam intensity maintains its average value to within about one plate thickness of the edge. Alternatively, a small heater at the edge can be used to maintain temperature uniformity. It must deliver a very small power, $W A l$, where $A$ is the crystal surface area not filled by the laser pulses. This will cause the heat loading to remain uniform out to the edge and eliminate any temperature drop there.

We conclude that there are no extra beam quality requirements for high average power harmonic generation beyond those given above for single pulse.

\section{Turbulent Dephasing}

The effect of random fluctuations on light propagation has been studied by several authors [7], [49], [20]. In propagating through the layers, a part of the beam is scattered by the turbulence and the rest passes on unperturbed [20]. The unscattered portion of the beam is $\exp \left(-s^{2}\right)$, were $s$ is the rms phase acquired in the layer:

$$
s=K_{o} b\left(n_{o}-1\right)\langle\delta \rho / \rho\rangle
$$

where $b$ is the channel width, $n_{o}$ is the refractive index at the mean mixed density, and $k_{o}=2 \pi / \lambda$. The beam divergence of the scattered wave is

$$
\theta=A\left(n_{o}-1\right)\langle\delta \rho / \rho\rangle\left(l_{s} b / l_{t}^{2}\right)^{1 / 2} .
$$

Here, $l_{s}$ is the longitudinal correlation length, $l_{t}$ is the transverse correlation length, and $\delta \rho / \rho$ is the fluctuation of the density in the turbulent gas. $\boldsymbol{A}$ is a numerical factor of order unity, depending on the statistics and mean distribution of the density.

The flow patterns in the turbulent boundary layer have been studied using Schlieren methods to photograph the gas density [16], [43]. The experiments were carried out under adiabatic conditions, where the gas carried no heat 
away from the wall. Under these conditions the density fluctuations are proportional to the turbulence intensity $\delta \rho / \rho=M^{2} \delta u / u$ where $M$ is the Mach number. The velocity fluctuation reaches a maximum at the edge of the boundary layer. It is also largest for Reynolds numbers in the range 2000-4000. The maximum value is about 0.1 , and the mean fluctuation across the flow channel is about 0.05 . The density fluctuations are therefore bounded by 0.1 for subsonic flow, and are probably somewhat less:

$$
0.1>\delta \rho / \rho>0.01 \text {. }
$$

Note that the turbulent Prandtl number in a gas is close to unity. Therefore the thermal contributions to $\delta \rho / \rho$ are expected to be of the same order as the momentum contribution.

The turbule size in the wall region is proportional to the distance from the wall. In the wake region, the turbule size varies little with location in the flow channel. It can also be shown that the shear forces in the boundary layer give rise to a longer correlation length parallel to the flow direction than perpendicular to the wall. We may therefore estimate $l_{t} \sim b$ and $l_{s} \sim c b$ where $c$ is a numerical constant approximately equal to the ratio of the wall region thickness to the channel width, and is typically about 0.1 .

Using the above estimates for the maximum density fluctuation and the scale lengths, the turbulent dephasing is typically less than $0.1 \mathrm{mrad} /$ atm for nitrogen, and 0.01 $\mathrm{mrad} / \mathrm{atm}$ for helium. Estimates for other gases can be scaled from this using the data in Table I. The divergence for $N-1$ channels is

$$
\theta_{N}=\theta(N-1)^{1 / 2}
$$

The total beam divergence is therefore about $0.1-1 \mathrm{mrad}$ for nitrogen, and $0.01-0.1 \mathrm{mrad}$ for helium, at $1 \mathrm{~atm}$.

The effect of turbulent dephasing on harmonic generation is most easily expressed in terms of the beam quality parameter, $Q$. This is increased by turbulence by an amount which depends on the beam dimension $H$

$$
Q^{\prime}=Q+\theta_{N} H / \lambda \text {. }
$$

The deleterious effects of the turbulence are therefore minimized by designing to small apertures.

\section{Thermal Stress in Thin Plates}

In an anisotropic medium the relation between stress and strain is [5]

$$
\eta_{i j}=s_{i j k l} S_{k l}+\alpha_{i j} T
$$

where $s$ is the elastic compliance tensor of the crystal and $T$ is its temperature. In the usual stress formulation of a thermoelastic problem, the stress field must satisfy both the equations of equilibrium of the solid and also the socalled compatibility equations. The equations of equilibrium are

$$
S_{i j, j}=0
$$

where ",$j$ " indicates differentiation with respect to $x_{j}$. The compatibility equations are due to the form of the strain tensor. The strain-displacement relation is

$$
\eta_{i j}=u_{i, j}+u_{j, i} \text {. }
$$

Operating on the strain tensor with antisymmetric derivatives (curl operators) gives

$$
\epsilon_{i j k} \epsilon_{r s t} \eta_{k t, j s}=0
$$

where $\epsilon_{i j k}$ is the fully antisymmetric tensor of rank three. Equation (58) is an identity satisfied by the strain tensor. On inserting the generalized Hooke's law into (58) the compatibility equations for the stresses are obtained:

$$
\epsilon_{i j k} \epsilon_{r s t}\left(s_{k t m n} S_{m n, j s}+\alpha_{k t} T_{, j s}\right)=0 .
$$

There are three equilibrium equations (56) and six compatibility equations (59), giving nine equations for the nine stress variables.

The stress formulation is completed by specifying the normal component of the stress on the boundary

$$
S_{i j} n_{j}=G_{i} \text {. }
$$

In many problems, the applied stress $G$ is zero.

To apply this to the current problem, Cartesian axes are defined in which $z$ is the beam direction and is, of course, perpendicular to the crystal surfaces, which therefore define the $(x, y)$ plane. The $(x y z)$ frame is the laboratory frame and is related by a three-dimensional rotation to either the optical $(\alpha \beta \gamma)$ frame or the crystallographic $(X Y Z)$ frame. In the thin-plate geometry we take the temperature distribution to be independent of $x$ and $y$, and therefore we are looking for a solution in which the stresses are also independent of $x$ and $y$ [17], [18]. Then, the equations of equilibrium, together with the boundary conditions, imply that all the stress components are zero except $S_{x x}, S_{x y}$ and $S_{y y}$. After some algebra the compatibility equations become

$$
\begin{aligned}
& s_{x x m n} S_{m n}^{\prime \prime}+\alpha_{x x} T^{\prime \prime}=0 \\
& s_{x y m n} S_{m n}^{\prime \prime}+\alpha_{x y} T^{\prime \prime}=0 \\
& s_{y y m n} S_{m n}^{\prime \prime}+\alpha_{y y} T^{\prime \prime}=0
\end{aligned}
$$

where prime denotes differentiation with respect to $z$. The sum over $m n$ is restricted to the terms $(x x),(x y)$, and ( $y y$ ), because all other stresses are zero. These are therefore three linear equations for the three remaining stresses in terms of the given temperature distribution, $T$. Assuming that the determinant of the appropriate compliances is not zero, the solution is obtained by standard linear algebra and is linear in $T^{\prime \prime}$ :

$$
S_{m n}^{\prime \prime}+\xi_{m n} T^{\prime \prime}=0 .
$$

The solution of (64) is

$$
S_{m n}=-\xi_{m n} T+A_{m n}+B_{m n} z
$$

where the constants $A$ and $B$ are to be determined from the boundary conditions. A good approximation in thin 
plates is to choose the constants to give zero force and moment over the edges of the plate. Then

$$
S_{m n}=\xi_{m n}\left[-T(z)+U_{0}+z U_{1}\right]
$$

where

$$
\begin{aligned}
& U_{0}=(1 / l) \int_{0}^{l} T\left(z^{\prime}\right) d z^{\prime} \\
& U_{1}=\left(12 / l^{3}\right) \int_{0}^{l} z^{\prime} T\left(z^{\prime}\right) d z^{\prime} .
\end{aligned}
$$

Clearly, this approximation to the solution does not have completely traction-free edges. However, at distances from the edges greater than about one plate thickness, Saint Venant's principle implies that this solution is an accurate approximation even for traction-free boundary conditions. It clearly satisfies the boundary conditions on the planes $z=0$ and $z=l$; it is therefore a good approximation to the unique solution to the anisotropic thermoelastic problem.

For temperature distributions which are symmetric about the midplane $z=l / 2$, the moment term $U_{1}$ is zero. Also, the term $U_{0}$ is the mean temperature of the plate. Thus, for these conditions the stresses are proportional to the temperature difference $\Delta T$ :

$$
S_{m n}(z)=\xi_{m n} \Delta T(z) .
$$

The stresses in the plate surface are given by $\Delta T$ and $\xi_{m n}$, which is a function only of the 27 compliances and of the orientation of the crystallographic axes ( $X Y Z$ ) relative to the laboratory frame $(x y z)$. The general expression for the $\xi$ is very cumbersome; as an example, we will evaluate them below in Section V, for KD*P.

The principal thermal stresses in the crystal are transverse to the beam direction and are largest at the crystal surface. They are parallel to the crystal surface. The surface is, of course, not precisely flat but contains pits, scratches, and other optically invisible defects which normally do not affect optical propagation. However, these surface defects will grow to macroscopic proportions if the surface stress exceeds a certain threshold. In increasing its size, the defect releases strain in the surrounding material. The defect will grow if the energy required to increase the defect size is less than the energy released by the accompanying reduction in strain. If this occurs, the crystal will become crazed, or it will shatter altogether.

Each surface defect is characterized by its own unique threshold, which depends on the size and shape of the defect. Typically the threshold for a defect of size $a$ is

$$
S=S_{0} / \sqrt{ } a
$$

where $S_{0}$ is the fracture toughness. Crystal failure occurs when the surface stress exceeds the lowest of all the defects' thresholds; according to (70), this is the threshold for the largest defect. In general, the surface stress is proportional to the maximum temperature difference in the crystal and is independent of its thickness.
The surface experiences three stresses, two of them tensile and one shear. It may also have an anisotropic strength. The surface will fracture if one of the stresses exceeds the surface strength for that direction and stress type. To avoid fracture the longitudinal temperature rise must not exceed the smallest of these thresholds. The anisotropy in fracture temperature can be large; in $\mathrm{KD}^{*} \mathrm{P}$ its variation with the orientation of the crystal surface is about a factor of six.

\section{Stress Optics in Thin Plates}

The stress-optic effect is described by a tensor which relates the perturbation in the inverse of the dielectric tensor $(1 / \epsilon)$ to the local stresses [36]

$$
\Delta(1 / \epsilon)_{i j}=q_{i j k l} S_{k l} \text {. }
$$

The two electromagnetic wave modes in the crystal have indexes $n_{a}$ and $n_{b}$, and polarizations $a_{i}$ and $b_{i}$, in the absence of stresses. These vectors are perpendicular to the beam direction and therefore lie in the crystal surface. The local stresses perturb these modes by changing their indexes and rotating the eigenmodes. The perturbations in the refractive indexes are

$$
\begin{aligned}
& \Delta\left(1 / n_{a}^{2}\right)=a_{i} a_{j} q_{i j k l} S_{k l} \\
& \Delta\left(1 / n_{b}^{2}\right)=b_{i} b_{j} q_{i j k l} S_{k l}
\end{aligned}
$$

and the rotation angle is

$$
\chi=a_{i} b_{j} q_{i j k l} S_{k l} /\left(1 / n_{a}^{2}-1 / n_{b}^{2}\right) .
$$

The stresses are given by the solution of the thermoelastic problem, $S_{k l}=\xi_{k l} \Delta T$. Thus the stress-optic effects are linear in the longitudinal temperature rise.

The rotation of the eigenvectors due to the stresses is essentially the ratio of the stress-induced birefringence to the natural birefringence along the given direction of propagation, $\vec{k}$. The induced birefringence is small for most media, around $10^{-5}$ or $10^{-6}$. Except for propagation close to an optic axis, the natural birefringence is much larger, around $10^{-2}$, and the fraction of the energy rotated into the other polarization is less than $10^{-6}$. Therefore, we ignore the stress-induced polarization rotation in all harmonic generation media. We note in passing, however, that this is not always possible for other media, such as laser host materials or electrooptic media.

There is an important sum rule concerning the phases accumulated by the two polarization states in completely traversing the crystal. For each stress component the integral over the crystal depends only on the moment of the temperature distribution. For example

$$
\int_{0}^{l} S_{a a} d z=\xi_{a a} U_{1} l^{2} / 2
$$

For symmetric temperature distributions, the moment is zero, and consequently the phase accumulated due to 
stress-induced index changes is also zero:

$$
\int_{0}^{l} \delta n_{a} d z=\int_{0}^{l} \delta n_{b} d z=0
$$

This shows that stress-induced index changes do not accumulate from one crystal to the next. They affect only the phase-matching condition in a single plate, just as the temperature profile does. This is the real justification for including both direct temperature dephasing and the indirect effect through the induced stresses in the single parameter $\beta_{t}$. In fact, ignoring the rotation of the eigenvectors

$$
d n_{a} / d T=\partial n_{a} / \partial T-(1 / 2) n_{a}^{3} a_{i} a_{j} q_{i j k l} \xi_{k l}
$$

where $\xi_{k l}$ is taken from the solution to the thermoelastic problem, (61)-(63). There is an analogous equation for the other index.

\section{Thermal Stress in KD*P Plates}

In second-harmonic generation from 1064 to $532 \mathrm{~nm}$ in a type II scheme, the crystal is oriented so that the 1064 $\mathrm{nm}$ drive makes an angle $\theta$ with the $Z$-axis, as in Fig. 6 . In the crystallographic frame, the beam direction is

$$
\vec{k}=(\sin \theta \cos \phi, \sin \theta \sin \phi, \cos \theta) .
$$

The angles $\theta$ and $\phi$, shown in Fig. 6 are

$$
\theta=54^{\circ}, \phi=45^{\circ} \text {. }
$$

The surface of the crystal is perpendicular to $\vec{k}$. We can take the laboratory $x$ - and $y$-directions to be the polarization vectors for the ordinary and extraordinary ray in the crystal. Thus

$$
\vec{x}=\vec{o} ; \vec{y}=\vec{e} .
$$

It is now straightforward to evaluate the relevant piezoelastic constants in the laboratory frame. For example, in terms of Cartesian components in the $(X Y Z)$ frame the $x x x x$ component in the laboratory frame is

$$
s_{x x x x}=o_{i} o_{j} s_{i j k l} o_{k} o_{l} .
$$

With these formulas, and similar expressions for the thermal expansion and thermal conductivity tensors, the parameters $\xi_{m n}$ which determine the thermal stresses can be evaluated. It is immediately clear that the $(x y)$ shear stress is zero. That is

$$
s_{x y x y} S_{x y}^{\prime \prime}+\alpha_{x y} T^{\prime \prime}=0
$$

and $\alpha_{x y}=0$. Therefore, there are no shear forces introduced by the thermal gradients

$$
\xi_{x y}=0 .
$$

The two remaining parameters are

$$
\xi_{x x}=\frac{-\alpha_{x x} s_{y y y y}+\alpha_{y y} s_{x x y y}}{s_{x x y y}^{2}-s_{x x x x} s_{y y y y}}=0.325 \mathrm{MPa} / \mathrm{K}
$$

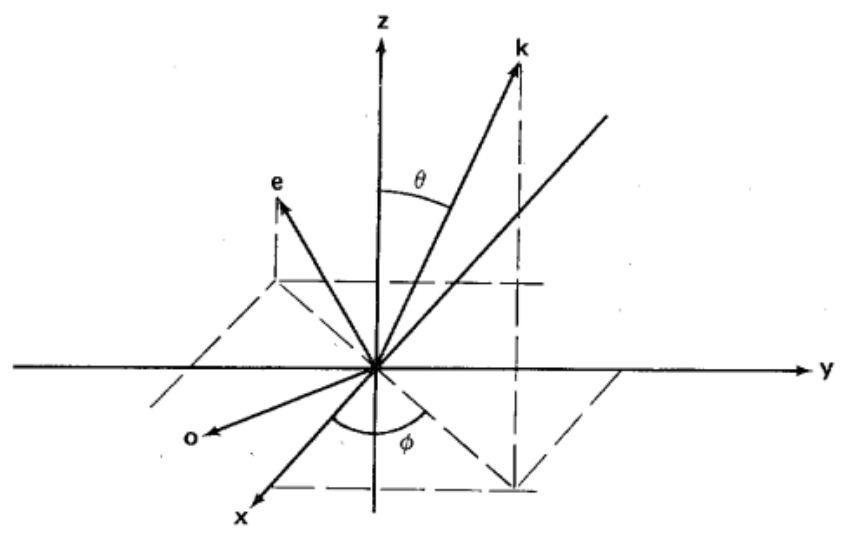

Fig. 6. The $o$ - and $e$-vectors associated with a ray with wavevector $\vec{k}$ in an anisotropic crystal. In uniaxial media, these are the polarizations of the two eigenmodes of electromagnetic waves in the crystal. In biaxial media, the eigenpolarizations are rotated relative to the $(o e)$ basis set around the $k$-vector. The (oe) vectors lie in the crystal surface if the ray enters normally and provide a useful basis set for thermooptic and stressoptic calculations such as those in Section V.

and

$$
\xi_{y y}=\frac{\alpha_{x x} s_{x x y y}-\alpha_{y y} s_{x x x x}}{s_{x x y y}^{2}-s_{x x x x} s_{y y y y}}=0.565 \mathrm{MPa} / \mathrm{K}
$$

where we have used the data in Table II.

The fracture toughness of $\mathrm{KD} * \mathrm{P}$ was measured recently [37]

$$
S_{o}=0.09 \mathrm{MPa} \cdot \mathrm{m}^{1 / 2} .
$$

For an assumed surface defect size of $0.1 \mathrm{~mm}$, the surface strength is $9 \mathrm{MPa}$. The maximum temperature difference is that for which the crystal would fracture in the extraordinary direction

$$
\Delta T_{f}=16 \mathrm{~K} .
$$

The stress-optic contribution to the thermal dephasing is straightforwardly evaluated using the constants in Table II. In the absence of stress-optic data for $\mathrm{KD} * \mathrm{P}$ we used the data for KDP. In KD*P, which is uniaxial, the $(a b)$ basis set is the $(o e)$ basis set in the surface. We find

$$
\begin{aligned}
& \frac{\partial n_{o}}{\partial S} \frac{d S}{d T}=-1.910^{-6} /{ }^{\circ} \mathrm{C} \\
& \frac{\partial n_{e}}{\partial S} \frac{d S}{d T}=-3.1410^{-6} /{ }^{\circ} \mathrm{C}
\end{aligned}
$$

These are to be compared with the stress-free derivatives

$$
\begin{aligned}
& \frac{\partial n_{o}}{\partial T}=-28.610^{-6} /{ }^{\circ} \mathrm{C} \\
& \frac{\partial n_{e}}{\partial T}=-20.010^{-6} /{ }^{\circ} \mathrm{C} .
\end{aligned}
$$

The stress contributes to the temperature derivative of the birefringence with the same sign, and might be expected to increase the temperature sensitivity by about 15 
TABLE II

MATERIAL CONSTANTS FOR KD*P

\begin{tabular}{llll}
\hline \hline Nonlinear coefficient & (type II, $1.064 \mu \mathrm{m})$ & $C$ & $0.97 \mathrm{GW}^{-1 / 2}$ \\
Angular sensitivity & (type II, $1.064 \mu \mathrm{m})$ & $\beta_{o}$ & $2.510^{5} \mathrm{~m}^{-1} / \mathrm{rad}$ \\
Temperature sensitivity & $\beta_{I}$ & $47 \mathrm{~m}^{-1} / \mathrm{K}$ \\
Absorption $(1.064 \mu \mathrm{m})$ & $\alpha_{o}$ & $1.2 \mathrm{~m}^{-1}$ \\
\multicolumn{1}{c}{$(0.523 \mu \mathrm{m})$} & $\alpha_{e}$ & $<0.1 \mathrm{~m}^{-1}$ \\
& $\alpha_{o}, \alpha_{e}$ & $<0.1 \mathrm{~m}^{-1}$ \\
Thermal conductivity & $K_{11}$ & $1.86 \mathrm{~W} / \mathrm{m} / \mathrm{K}$ \\
& $K_{33}$ & $2.09 \mathrm{~W} / \mathrm{m} / \mathrm{K}$ \\
Thermal expansion & $\alpha_{11}$ & $2.4910^{-5}$ \\
& $\alpha_{33}$ & $4.4010^{-5}$ \\
Surface toughness & $S_{o}$ & $0.09 \mathrm{MPa} \cdot \mathrm{m}^{-1 / 2}$ \\
Compliance tensor & $S_{11}$ & $15.8(\mathrm{TPa})^{-1}$ \\
& $S_{12}$ & $2.1(\mathrm{TPa})^{-1}$ \\
& $S_{13}$ & $-4.0(\mathrm{TPa})^{-1}$ \\
& $S_{33}$ & $20.1(\mathrm{TPa})^{-1}$ \\
& $S_{44}$ & $79.0(\mathrm{TPa})^{-1}$ \\
& $S_{66}$ & $168(\mathrm{TPa})^{-1}$ \\
Stress-optic tensor ${ }^{\mathrm{a}}$ & $q_{11}$ & $4.15(\mathrm{TPa})^{-1}$ \\
& $q_{12}$ & $4.08(\mathrm{TPa})^{-1}$ \\
& $q_{13}$ & $1.12(\mathrm{TPa})^{-1}$ \\
& $q_{31}$ & $3.60(\mathrm{TPa})^{-1}$ \\
& $q_{33}$ & $0.44(\mathrm{TPa})^{-1}$ \\
& $q_{44}$ & $-1.47(\mathrm{TPa})^{-1}$ \\
& $q_{66}$ & $-10.26(\mathrm{TPa})^{-1}$ \\
\hline${ }^{2}$ Data reported here are for KDP. $1 \mathrm{TPa}=10^{12} \mathrm{~Pa}, 1 \mathrm{MPa}=10^{6} \mathrm{~Pa}$.
\end{tabular}

percent. Exact calculations require the dispersion of the thermooptic coefficients.

In this way all of the parameters are determined at the standard crystal orientation for harmonic generation. Other things being equal, fracture resistance is greatest when the ratio $\xi / K_{z z}$ is smallest. The phase-matching condition allows $\phi$ to be adjusted a little, but not $\theta$. However, the nonlinear coupling $C$ is proportional to $\sin (2 \phi)$, and the performance will deteriorate if $\phi$ deviates from 45 deg.

It is interesting to note that the fracture limit is anisotropic crystals depends on the material constants for specific directions in the crystal. It is sometimes possible, by adjusting the composition of the crystal, to arrange for zero thermal expansion along certain directions. It is also sometimes possible to directionally adjust the thermal conductivity. In particular, a material with zero expansion perpendicular to the beam (i.e., in the crystal surfaces) will generate no thermal stress. There are several known acentric materials whose thermal expansion can be made to vanish or be slightly negative. These include the $\mathrm{Eu}-$ cryptite-Spodumene class and a new class CTP (calcium titanium phosphate) [53], of which NaYSiO4 is an example and is acentric. These possibilities may eventually be exploited to engineer materials with higher resistance to thermal fracture.

\section{System Design}

High conversion efficiency clearly requires that the total dephasing be constrained. High average power requires (among other things) that a sufficient portion of the total dephasing budget be available to thermal dephasing.

The total dephasing includes the effects of the coolant dispersion, the beam divergence and bandwidth, the longitudinal temperature profile, the transverse temperature profile, and index inhomogeneities in the crystal. Thus

$$
\delta=\delta_{g}+\delta_{\text {tr }}+(1 / 8) \delta_{T}+\delta_{\theta}+\delta_{\lambda}+\delta_{n}
$$

where

$$
\begin{aligned}
& \delta_{g}=l_{s} / l_{c} \\
& \delta_{\theta}=\beta_{\theta} \Delta \theta N l \\
& \delta_{T}=\beta_{t} \Delta T l \\
& \delta_{\text {tr }}=\beta_{t} \Delta T_{\text {tr }} N l \\
& \delta_{\lambda}=\beta_{\lambda} \Delta \lambda N l \\
& \delta_{n}=2 \pi \Delta n \cdot N l / \lambda
\end{aligned}
$$

and the temperature differences are

$$
\begin{aligned}
\Delta T & =\alpha_{I} I_{\mathrm{av}} l^{2} / 8 K \\
\Delta T_{\mathrm{tr}} & =\Delta T\left(8 K / C_{p} v l_{s}\right)(H / l) .
\end{aligned}
$$

To achieve high efficiency (Fig. 1) the total dephasing $\delta$ must be constrained:

$$
\delta<\pi / 10
$$

The total dephasing is the parameter which controls the conversion efficiency of the stack of $\mathrm{N}$-plates. Thus most of the contributions to $\delta$ are proportional to the total crystal thickness, $N \cdot l$. However, the longitudinal thermal dephasing and the contribution of the dispersion of the coolant gas are proportional to the individual plate thickness, $l$. The reason is that these are reset to zero at the entrance face of each plate, whereas the others cannot be reset.

The angular dephasing term is determined by the requirement of high conversion efficiency. The laser and 
crystal parameters must be matched so that the condition for high efficiency is satisfied. This condition is (19). We define a threshold power as follows:

$$
P_{\text {th }}=\left(\beta_{\theta} \lambda / C\right)^{2}
$$

then

$$
\eta_{o} / 4 \delta_{\theta}^{2}=P / P_{\mathrm{th}} Q^{2} \cdot\left(H / H^{\prime}\right)
$$

where $Q$ is the etendu in the sensitive direction of the crystal, and $P$ is the peak power (not intensity). High efficiency will be obtained for $\eta_{o}$ greater than about 2 . From the definition of $\eta_{o}$, the total crystal length $(N \cdot l)$ is given by

$$
(N \cdot l)^{2} / H H^{\prime}=\eta_{o} / C^{2} P .
$$

In accordance with our prior arguments, this condition fixes the crystal shape, but not its size; the crystal size is chosen to reduce the fluence below the damage limit. For fixed $\eta_{o}$, the angular dephasing is determined by (102). Clearly the angular dephasing is minimized by using a material whose threshold power is also minimal. It is not enough that the laser match the crystal's threshold power; high average power at high conversion efficiency requires that the laser peak power exceed $P_{\text {th }}$ by a significant ratio, to minimize the angular dephasing and leave the major part of the dephasing budget to the thermal dephasing.

Table III lists the threshold powers, damage thresholds, and other average power figures of merit for some common materials for doubling $1064 \mathrm{~nm}$.

The dependence of the detuning $\delta$ on the plate spacing $l_{s}$ appears in the first two of the six terms in $\delta$. The coolant dispersion term is linearly proportional to $l_{s}$, whereas the transverse temperature term is inversely proportional to $l_{s}$. There is therefore an optimum plate separation which minimizes the total detuning. This is

$$
l_{s}^{2}=\left(\delta_{T} E m N\right) \cdot l_{c}^{2}
$$

where the parameter $E m$ is

$$
E m=512 K \kappa^{\prime} / C_{p} v l_{c} .
$$

Here, $k^{\prime}=H^{\prime} / l>1$ is the aspect ratio in the direction of flow. Numerical values for $E m$ range from about 0.07, obtained for helium-cooled $\mathrm{KD} * \mathrm{P}$ at a Reynolds number of $10^{4}$, to about 0.8 , obtained for nitrogen-cooled $\mathrm{LiNbO}_{3}$. For this plate separation the total dephasing is

$$
\delta=(1 / 4)\left(\delta_{T} E m N\right)^{1 / 2}+(1 / 8) \delta_{T}+\delta_{\theta}+\delta_{\lambda}+\delta_{n} \text {. }
$$

The dephasing constraint limits $\delta$ and therefore also limits $\delta_{T}$ and the average power. The maximum dephasing available to $\delta_{T}$ within the dephasing budget is

$$
\delta_{T 0}=8\left(\delta-\delta_{\theta}-\delta_{\lambda}-\delta_{n}\right) .
$$

Transverse effects diminish this further, depending on the value of the transverse figure of merit, $\mathrm{Em}$. That is

$$
\delta_{T} / \delta_{T 0}=\left[(1+v)^{1 / 2}-v^{1 / 2}\right]^{2}
$$

TABLE III

Material Figures of Merit For Doubling $1.064 \gamma \mathrm{m}$

\begin{tabular}{lccrc}
\hline \hline \multicolumn{1}{c}{ Material } & $P_{\text {th }}(\mathrm{MW})$ & $J_{o}\left(\mathrm{~kJ} / \mathrm{m}^{2}\right)$ & $W m(\mathrm{~W} \cdot \mathrm{mm})$ & $\Delta T_{F}\left({ }^{\circ} \mathrm{C}\right)$ \\
\hline $\mathrm{BaB}_{2} \mathrm{O}_{4}$ & 430 & 150 & 20000 & 90 \\
$\mathrm{KD}^{* P}$ & 67 & 70 & 500 & 16 \\
$\mathrm{LiIO}_{3}$ & 135 & 10 & 6120 & 15 \\
$\mathrm{MgO}^{2} \mathrm{LiNbO}_{3}$ & $n c p m$ & 100 & 400 & 3.8 \\
$\mathrm{KTP}$ & 0.05 & {$[100]^{\mathrm{a}}$} & {$[11000]$} & \\
$\mathrm{CD}{ }^{*} \mathrm{~A}$ & $n c p m$ & 70 & {$[100]$} & \\
\hline
\end{tabular}

${ }^{2}$ Quantities in brackets are estimates.

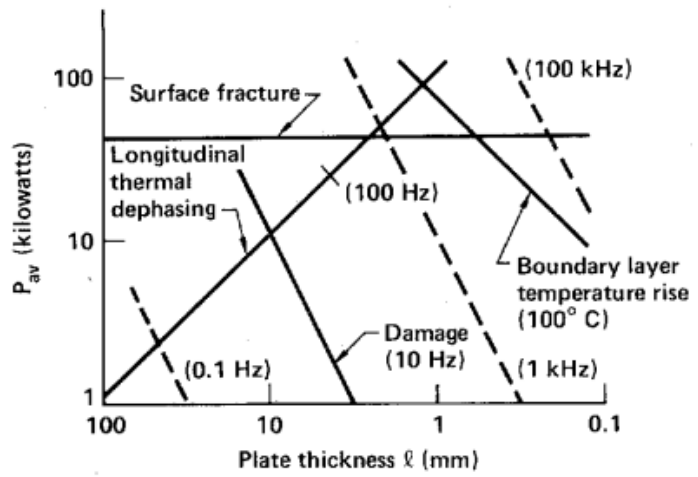

Fig. 7. The average power in a single crystal segment of KD*P doubling $1.064 \mu \mathrm{m}$. The choice of plate thickness and the average power depend on the tradeoff between thermal dephasing, damage, surface fracture, and cooling rate. The surface fracture limit is shown for surface defects $100 \mu \mathrm{m}$ in size, and the damage limit for $0.1,10,10^{3}$, and $10^{5} \mathrm{~Hz}$. All aspects of high average power design can be understood from the behavior of the lines on this graph, as system variables such as the gas flow velocity are varied.

where $v=E m \cdot \delta_{T 0} \cdot N$. The average power is limited by thermal dephasing to

$$
P_{\mathrm{av}}=8 K \delta_{T} \kappa \kappa^{\prime} / \alpha_{I} \beta_{t} l .
$$

The parameter $E m$ is the ratio of the thermal resistance of the crystal plates and the coolant gas, using the optical coherence length to determine the width of the flow channel. It is a thermooptical property of the flow, analogous to the Prandtl number of convective transport theory. Em determines the dependence of $\delta_{T}$ on $\delta$, and the relative importance of the transverse contributions to the thermal dephasing, and is a transverse figure of merit. For high $E m>>1$, the plate separation is the most important parameter in the dephasing; this is associated with the coolant dispersion and the transverse temperature rise. For low $E m<<1$, the longitudinal temperature rise is dominant. For all gases except helium, and most nonlinear crystals, $\mathrm{Em}$ will be in the high to moderate regime. However, for helium-cooled KD*P, Em is low and the longitudinal temperature rise dominates.

The system constraints controlling the average power are the cooling rate, surface fracture, thermal dephasing, and optical damage. In Fig. 7, the first three constraints are plotted against the inverse of the thickness of a single plate, $1 / l$, for $\mathrm{KD} * \mathrm{P}$ doubling $1064 \mathrm{~nm}$. These constraints are as follows. 
TABLE IV

TABLE OF SYMBOLS

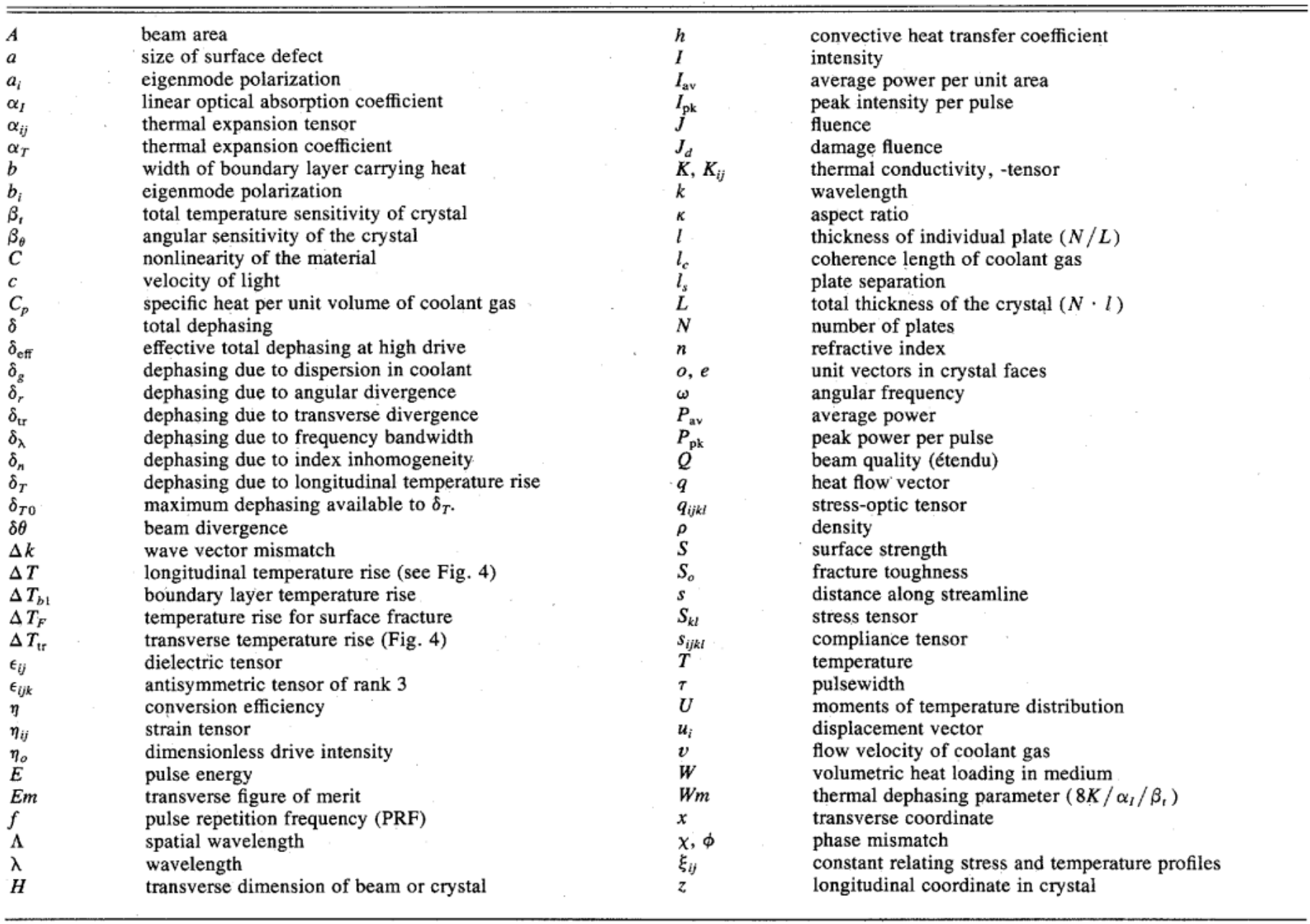

Thermal Dephasing:

$$
P_{\mathrm{av}}=\delta_{T}\left(\frac{8 K \kappa^{2}}{\beta_{t} \alpha_{I}}\right)(1 / l)
$$

Surface Fracture:

$$
P_{\mathrm{av}}=\frac{8 K \Delta T_{F} \kappa^{2}}{\alpha_{I}} .
$$

Cooling Rate:

$$
P_{\mathrm{av}}=\frac{2 h \Delta T_{b 1} \kappa^{2}}{\alpha_{I}}(l)
$$

Here, $\kappa^{2}=\kappa \kappa^{\prime}$, the ratio of beam area to $l^{2}$ and is taken to be $15^{2}=225$ for a brittle material like KD*P. The slopes of the average power constraints are $-1,0$, and 1 for the surface temperature, surface fracture, and thermal detuning respectively. The only system variable which affects these bounds is $N$, the number of plates, and it affects only the thermal dephasing bound for high $\mathrm{Em}$. The thermal dephasing is the most severe constraint for thick plates, and for thin plates the cooling rate limit is the most restrictive. In the intermediate region, the surface fracture limit may or may not be relevant, depending on the material parameters and cooling rate. For $\mathrm{KD} * \mathrm{P}$, it is apparently more restrictive than the other two constraints. The plate thickness at the intersections of the surface fracture and cooling rate bound is

$$
l_{1}=(4 K / h)\left(\Delta T_{F} / \Delta T_{s}\right)
$$

and the surface fracture and thermal detuning bounds intersect at

$$
l_{2}=\delta_{T} / \beta_{t} \Delta T_{F}
$$

and the thermal detuning and surface temperature bounds intersect at

$$
\begin{aligned}
l_{3} & =\left(l_{1} l_{2}\right)^{1 / 2} \\
& =2\left(\delta_{T} K / h \beta_{t} \Delta T_{s}\right) 1 / 2 .
\end{aligned}
$$

The surface fracture limit applies only if

$$
l_{1}<l_{2}
$$


and for this situation represents the maximum average power per aperture. The average power is independent of $l$ for $l_{1}>l>l_{2}$. This gives a range of $l$ for which $P_{\mathrm{av}}$ is maximum. Because the mechanical strength decreases with $1 / l$, the more robust designs have the thickest plates. The optimum plate thickness therefore occurs at the intersection of the thermal fracture boundary and the thermal dephasing boundary. That is, the plate thickness is $l_{1}$, and the optimum average power per aperture is

$$
P_{\max }=8 K \Delta T_{F} \kappa^{2} / \alpha_{l} .
$$

On the other hand, for tough materials the surface fracture limit is not relevant. The optimum plate thickness is $l_{3}$, and the maximum average power occurs for a unique plate thickness. Then the average power is

$$
P_{\max }=\left(4 \kappa^{2} / \alpha_{I}\right)\left(K h \Delta T_{s} \delta_{T} / \beta_{t}\right)^{1 / 2} .
$$

According to Fig. 7, the appropriate limit for $\mathrm{KD} * \mathrm{P}$ is the stress fracture limit. The surface fracture limit is about 45 $\mathrm{KW}$, whereas the surface temperature limit is about 90 $\mathrm{KW}$. The intersections are at $l_{1}=0.6 \mathrm{~mm}, l_{2}=2.5 \mathrm{~mm}$, and $l_{3}=1.1 \mathrm{~mm}$, so the appropriate plate thickness is $l_{2}$, and its aperture is $37.5 \mathrm{~mm}$.

We have already seen that on a single-pulse basis the problem of optical damage is solved by scaling up the crystal and beam dimensions. However, thermal effects are increased as the crystal size increases; therefore, maximum average power in a single aperture is obtained with the smallest crystal dimensions compatible with avoiding optical damage. For fixed pulse energy, this is equivalent to a maximum pulse repetition frequency (PRF). At fixed average power, increasing the PRF reduces the pulse energy, and at some PRF, the pulse energy is so small that damage is no longer an issue. In this way, the role of the damage threshold is to define the PRF below which the system is damage limited, and above which it is limited by the cooling rate or fracture limits. The average power can be expressed in terms of the fluence per pulse:

$$
P_{\mathrm{av}}=f J_{d}(\kappa l)^{2} .
$$

Then inserting the limit on the average power, $P_{\max }$, into (120) we obtain a limiting PRF $f_{0}$ :

$$
\begin{aligned}
f & >f_{0}(l) \\
& =P_{\max } /\left(l \kappa^{2}\right) J_{d}(\tau) .
\end{aligned}
$$

Here, $l$ is either $l_{1}$ or $l_{3}$, whichever is the appropriate plate thickness for the system. At repetition rates below $f_{0}$, the average power is determined by optical damage and not by the power-handling limits of the crystal and coolant. To illustrate this, the damage limit is shown in Fig. 7 for repetition rates of $0.1 \mathrm{~Hz}, 10 \mathrm{~Hz}, 1 \mathrm{kHz}$, and $100 \mathrm{kHz}$. Its slope is -2 , and in general its location depends on $f$. At low PRF, the optimal plate thickness is given by the intersection of the damage limit with the thermal dephas- ing bound. (This is the situation in most average power experiments to date.) This plate thickness is

$$
l_{4}=\left(8 K \delta_{T} / \beta_{i} \alpha_{I} J_{d} f\right)^{1 / 3} .
$$

As $f$ increases, the damage limit moves upward, allowing greater average power, and $l_{4}$ decreases. At a sufficiently high repetition rate, $l_{4}$ is smaller than the larger of $l_{3}$ or $l_{1}$, and the average power is limited by the thermal efficiency rather than the optical damage threshold. At this point, the system has changed from essentially a singlepulse system operated so as to deliver several pulses per second, to a true average power system. In this sense, the damage limit defines the boundary between single-pulse and average power systems. According to Fig. 7, this transition occurs for $\mathrm{KD} * \mathrm{P}$ at a PRF of a few kilohertz.

\section{Conclusions and Summary}

The purpose of this paper was to assess solid-state nonlinear materials for high average power applications. The current state of the art in high average power is a few tens of watts. It has been shown here that solid-state nonlinear materials can in fact surpass the current state of the art by at least three orders of magnitude. Moreover, configurations exist which permit essentially unconstrained scaling of average power.

In average power operation the linear optical absorption in the crystals causes temperature gradients which can reduce the conversion efficiency by disturbing phase matching. If nonlinear crystals were perfectly transparent, there would be no special aspects to average power harmonic generation. Design would then proceed along the lines outlined in Section II, and average power would cease to be an issue. However, most nonlinear materials are not sufficiently transparent that thermal effects can be ignored. An absorption of $0.1 \mathrm{~m}^{-1}$ is often large enough to be significant.

Because the interest is in high average power, intrinsically unscalable geometries such as tight focusing were not considered. A careful assessment of the heat flow in the nonlinear crystal showed that the thermal gradients must be perpendicular to the crystal faces for efficient, scalable heat removal. The optimum geometry uses plane waves and a number of crystal plates with a coolant gas flowing between them to remove the heat deposited as a result of optical absorption. The gas flow is high velocity and turbulent for efficient heat removal. By dividing a large aperture into segments (which was successfully demonstrated on NOVA [46]), and optimizing each segment, the total average power in the entire aperture is essentially unconstrained. Each segment consists of several plates of single crystals through which the beam passes consecutively, as shown in Fig. 4. The average power in each segment is constrained by thermal dephasing, surface fracture, surface temperature, and optical damage. Each of these effects was analyzed in detail. It was found (see Fig. 7) that a single segment of a generator using 
$\mathrm{KD} * \mathrm{P}$ to double $1.064 \mu \mathrm{m}$, is ultimately capable of average powers approaching $100 \mathrm{KW}$. With a relatively modest number of segments, very high average powers can be envisioned for the total aperture.

It is worth noting that for doubling wavelengths below $1.064 \mu \mathrm{m}$, the absorption of $\mathrm{KD} * \mathrm{P}$ is considerably smaller than at $1.064 \mu \mathrm{m}\left(0.6 \mathrm{~m}^{-1}\right)$. Therefore, the ultimate average power per segment for doubling to the blue or UV regions is much higher than $100 \mathrm{KW}$. A precise value cannot be given without accurate absorption data; however, a value typical of transparent window materials, 0.03 $\mathrm{m}^{-1}$, permits the ultimate average power per segment to be greater than $1 \mathrm{MW}$.

The temperature gradients cause thermal dephasing of the waves, and disturb phase matching. There are two contributions to the temperature variation in the crystal (Fig. 5). One is the longitudinal temperature rise, associated with the flow of heat outwards to the crystal surfaces. The other is the transverse temperature rise, associated with the rise in temperature of the coolant as it traverses the crystal face. Now, the total dephasing budget is constrained by the requirement of high conversion efficiency, as described in Section II. It contains six terms. These are the longitudinal and transverse temperature rise, the optical dispersion in the coolant, the angular divergence and linewidth of the drive, and the index inhomogeneity in the crystals. Of these, only two depend on the separation between the plates, these are the optical dispersion in the coolant, and the transverse temperature rise. The separation between the plates is therefore uniquely determined by the tradeoff between these two terms. With the plate separation fixed the thermal part of the total detuning then includes only two terms; the longitudinal temperature rise and the optimized combination of the optical dispersion and transverse temperature rise terms. Because the average power is related to the longitudinal temperature rise, highest average power is obtained by minimizing the transverse terms. The transverse terms are given by the transverse figure of merit $\mathrm{Em}$ and the available thermal dephasing $\delta_{T 0}$. Referring back to Fig. 7, the effect of the transverse terms can be visualized as a downward translation of the thermal dephasing line.

Overall, the prognosis for nonlinear optics at high average power is very encouraging. The heat load in a nonlinear material with moderate optical absorption and nominal thermomechanical properties can be handled gracefully with turbulent gas cooling. This conclusion is not limited to second harmonic generation, but appears to generalize to any (optical energy conserving) process involving frequency conversion. If a nonlinear material with nominal properties is capable of high efficiency on a pulseby-pulse basis, then it will also scale to high average power.

\section{ACKNOWLEDGMENT}

The author wishes to thank J. Emmett and many other colleagues at LLNL for stimulating conversations.

\section{REFERENCES}

[1] G. R. Anstis, P. Chantikul, B. R. Lawn, and D. B. Marshall, "A critical evaluation of indentation techniques for measuring fracture toughness: I, direct crack measurements," J. Amer. Ceram. Soc., vol. 64 , pp. $533-543,1981$.

[2] J. A. Armstrong, N. Bloembergen, N. Ducuing, and P. S. Pershan, "Interactions between light waves in a nonlinear dielectric," Phys. Rev., vol. 127, pp. 1918-1939, 1962.

[3] J. D. Barry and C. J. Kennedy, "Thermooptical effects of intracavity $\mathrm{Ba}_{2} \mathrm{Na}\left(\mathrm{NbO}_{3}\right)_{5}$ on a frequency double Nd-YAG laser," IEEE $J$. Quantum Electron., vol. QE-11, pp. 575-579, 1975.

[4] H. E. Bennett, A. J. Glass, A. H. Guenther, and B. E. Newnam, Eds., "Laser-induced damage in optical materials," available from NBS.

[5] B. A. Boley and J. H. Weiner, Theory of Thermal Stresses. New York: Wiley, 1960.

[6] M. Born and E. Wolf, Principles of Optics. Elmsford, NY: Pergamon, 1970 , p. 678 .

[7] L. A. Chernov, Wave Propagation in a Turbulent Medium. New York: McGraw-Hill, 1960.

[8] P. Chemla, "Synchronization of the phases of the first and second harmonic frequencies in nonlinear non-absorbing crystals under the interaction of two waves," Optik, vol. 28, pp. 346-359, 1968.

[9] R. C. Eckardt and J. Reintjes, "Phase-matching limitations of high efficiency second harmonic generation," IEEE J. Quantum Electron., vol. QE-20, pp. 1178-1187, Oct. 1984.

[10] D. Eimerl, M. A. Summers, B. C. Johnson, J. D. Williams, and G. J. Linford, LLNL Laser Program Annu. Rep. 1981, UCRL 5002181, pp. 7-1-7-12, 1981.

[11] D. Eimerl, LLNL Laser Program Ann. Rep. 1983, UCRL-50021-83, pp. 6-69-6-74, 1983.

[12] $\frac{-}{-}$, in Proc. Int. Conf. Lasers '84, E. Corcoran, Eds., STS Press, 1984, pp. 557-559.

[13] J. L. Emmett, W. F. Krupke, and W. R. Sooy, "The potential of high average power solid state lasers," LLNL Rep. UCRL 53571, Sept. 1984.

[14] T. S. Fahlen and P. E. Perkins, "Material and medical applications using a $20 \mathrm{~W}$ frequency doubled Nd:YAG laser," in Proc. Conf. Laser Eng., Opt., CLEO '84, paper THCl.

[15] J. E. Geusic, H. J. Levinson, S. Singh, R. G. Smith, and L. G. van Uitert, "Continuous $0.532 \mu \mathrm{m}$ solid state source using $\mathrm{Ba}_{2} \mathrm{NaNb}_{5} \mathrm{O}_{15}$," Appl. Phys. Lett., vol. 12, pp. 306-310, 1968.

[16] J. Hinze, Turbulence. New York: McGraw-Hill, 1975.

[17] G. Horvay and J. S. Born, "Thermal stresses in rectangular strips," J. Appl. Mech., vol. 22, pp. 401-406, 1955.

[18] G. Horvay, "Some aspects of Saint Venant's principle," J. Mech., Phys. Solids, vol. 5, pp. 77-94, 1957.

[19] M. V. Hobden, "Phase-matched second harmonic generation in biaxial crystals," J. Appl. Phys., vol. 38, pp. 4365-4372, 1967.

[20] C. B. Hogge, R. R. Butts, and M. Burlakoff, "'Characteristics of phase aberrated nondiffraction-limited laser beams," Appl. Opt., vol. 13, pp. 1065-1070, 1973.

[21] D. Hon, "Electrooptical compensation for self-heating in CD*A during second harmonic generation," IEEE J. Quantum Electron., vol. QE-12, pp. 148-151, 1976.

[22] - " "Average power breakthrough in nonlinear processes in crystals," IEEE J. Quantum Electron., vol. QE-13, pp. 998-1000, Sept. 1977.

[23] D. Hon, H. Brusselbach, and E. Woodbury, in Proc. SPIE, San Diego, CA, Aug. 1977.

[24] D. Hon, "High average power efficient harmonic generation," in Laser Handbook 3. Amsterdam, The Netherlands: North-Holland, 1979 , ch. B2, pp. 421-484.

[25] J. F. Holzrichter, D. Eimerl, E. V. George, J. B. Trenholme, W. W. Simmons, and J. T. Hunt, "High power pulsed lasers," J. Fus. Energy, vol. 2, pp. 5-45, 1982.

[26] K. E. Hulme, "Nonlinear optical crystals and their applications," Rep. Prog. Phys., vol. 36, pp. 497-540, 1973.

[27] H. Ito, H. Naito, and H. Inaba, "Generalized study on angular dependence of induced second-order nonlinear optical polarizations and phase-matching in biaxial crystals (T/E)," J. Appl. Phys., vol. 46, no. 9, pp. 3992-3998, 1975.

[28] W. M. Kays and E. Y. Leung, "Heat transfer in annular passagesHydrodynamically developed turbulent flow with arbitrarily pre- 
scribed heat flux," Int. J. Heat Mass Transfer, vol. 6, pp. 537-557, 1963.

[29] W. M. Kays, Convective Heat and Mass Transfer. New York: McGraw-Hill, 1966.

[30] D. A. Kleinman, "Optical Harmonic Generation in Nonlinear Media," in Laser Handbook 2. Amsterdam, The Netherlands: North Holland, 1972, ch. E4, pp. 1229-1258.

[31] W. Koechner, Solid State Laser Engineering. New York: SpringerVerlag, 1976.

[32] R. M. Kogan and T. G. Crow, "A high brightness one Joule, frequency doubled Nd:YAG laser," IEEE J. Quantum Electron., vol. QE-13, 1977.

[33] F. Kreith, Principles of Heat Transfer. International Textbook, 1963 , p. 345

[34] W. F. Krupke, E. V. George, and R. A. Haas, "Advanced Lasers for fusion," in Laser Handbook 3. Amsterdam, The Netherlands: North Holland, 1979, pp. 627-752.

[35] S. K. Kurtz, "New nonlinear optical materials," IEEE J. Quantum Electron., vol. QE-4, pp. 578-584, 1968.

[36] Landolt-Börnstein, Tables Gp III, Crystal and Solid State Physics, vol. 11, pp. 495-744, 1979.

[37] J. A. Marion, LLNL, private communication.

[38] R. C. Miller, "Nonlinear optical properties of ferroelectric crystals," J. Phys. Soc. Japan, vol. 28, pp. S15-S20, 1968.

[39] D. Milam and C. Weinzapfel, unpublished.

[40] E. Moses, H. Brusselbach. D. Stovall, and D. T. Hon, in Proc. Soc. Opt. Quantum Electron. Conf. Lasers, Appl., Orlando, FL, 1978.

[41] R. U. Orlov, "Optical harmonic generation in biaxial crystals," Radiophysics, Quantum Electron., vol. 12, pp. 1056-1058, 1969.

[42] T. S. Fahlen and P. E. Perkins, private communication.

[43] H. Schichting, Boundary Layer Theory. New York: McGraw-Hill, 1962.
[44] Y. R. Shen, "Recent advances in nonlinear optics," Rev. Mod. Phys., vol. 48 , pp. 1-32, 1976.

[45] X. Shenguu, G. Jiarong, and Zh. Jiaju, "The calculation of the phasematching angles and the effective second-order nonlinear coefficients in biaxial crystals," Jiaotong Daxue Xuebao (China), vol. 1, pp. $37-$ 52,1982 .

[46] W. W. Simmons and R. O. Godwin, "Nova laser fusion facility: Design, engineering and assembly overview," J. Nucl. Tech./Fusion, vol. 4, pp. 8-14, 1983.

[47] R. G: Smith, "Theory of intracavity optical second harmonic generation," IEEE J. Quantum Electron., vol. QE-6, pp. 215-223, 1970.

[48] M. A. Summers, R. D. Boyd, D. Eimerl, and E. M. Booth, "A twocolor frequency conversion system for high power lasers," in Proc. IEEE/OSA Conf. Laser Eng., Opt. (CLEO), 1981, p. 30.

[49] V. I. Tatarski, Wave Propagation in Turbulent Medium. New York: Dover, 1967.

[50] S. Velsko and D. Eimerl, "Precise measurements of optical dispersion using a new interferometric technique," Appl. Opt., vol. 25, pp. 1344-1347, 1985.

[51] J. Warner, "Phase-matching for optical up-conversion with maximum angular aperture-Theory and practices,"'Opto-Electron., vol. 1, pp. 25-31, 1969.

[52] F. Zernike and J. Midwinter, Applied Nonlinear Optics. New York: Wiley, 1973.

[53] R. Roy, D. K. Agarwal, J. Alamo, and R. A. Roy, "“[CTP]: A new structural family of near-zero expansion ceramics," Mater. Res. Bull., vol. 19, pp. 471-477, 1984.

David Eimerl, for a photograph and biography, see p. 410 of the April 1987 issue of this JourNal. 